\title{
Pharmacological and non-pharmacological strategies for preventing postherpetic neuralgia: a systematic review and network meta-analysis
}

\author{
Junhyeok Kim ${ }^{1, *}$, Min Kyoung Kim ${ }^{1, *}$, Geun Joo Choi $^{1}$, Hwa Yong Shin $^{1}$, Beom Gyu Kim ${ }^{2}$, and Hyun Kang ${ }^{1}$ \\ 'Department of Anesthesiology and Pain Medicine, Chung-Ang University College of Medicine, Seoul, Korea \\ ${ }^{2}$ Department of Surgery, Chung-Ang University College of Medicine, Seoul, Korea
}

Received July 26, 2021

Revised August 25, 2021

Accepted August 29, 2021

Handling Editor: Francis S. Nahm

\section{Correspondence}

Hyun Kang

Department of Anesthesiology and Pain Medicine, Chung-Ang University College of Medicine, 84 Heukseok-ro, Dongjakgu, Seoul 06911, Korea

Tel: +82-2-6299-2571, 2579, or 2586

Fax: +82-2-6299-2585

E-mail: roman00@naver.com

*These authors contributed equally to this work and are co-first authors.

\begin{abstract}
Background: Postherpetic neuralgia (PHN) is a refractory complication of herpes zoster (HZ). To prevent PHN, various strategies have been aggressively adopted. However, the efficacy of these strategies remains controversial. Therefore, we aimed to estimate the relative efficacy of various strategies used in clinical practice for preventing PHN using a network meta-analysis (NMA).

Methods: We performed a systematic and comprehensive search to identify all randomized controlled trials. The primary outcome was the incidence of PHN at 3 months after acute $\mathrm{HZ}$. We performed both frequentist and Bayesian NMA and used the surface under the cumulative ranking curve (SUCRA) values to rank the interventions evaluated.

Results: In total, 39 studies were included in the systematic review and NMA. According to the SUCRA value, the incidence of PHN was lower in the order of continuous epidural block with local anesthetics and steroids (EPI-LSE), antiviral agents with subcutaneous injection of local anesthetics and steroids (AV $+s L S)$, antiviral agents with intracutaenous injection of local anesthetics and steroids (AV + iLS) at 3 months after acute HZ. EPI-LSE, AV + sLS and AV + iLS were also effective in preventing PHN at 1 month after acute $\mathrm{HZ}$. And paravertebral block combined with antiviral and antiepileptic agents was effective in preventing PHN at 1, 3, and 6 months.

Conclusions: The continuous epidural block with local anesthetics and steroid, antiviral agents with intracutaneous or subcutaneous injection of local anesthetics and a steroid, and paravertebral block combined with antiviral and antiepileptic agents are effective in preventing PHN.
\end{abstract}

Key Words: Anesthesia, Local; Anticonvulsants; Autonomic Nerve Block; Bayes Theorem; Injections, Epidural; Nerve Block; Network Meta-Analysis; Neuralgia, Postherpetic; Stellate Ganglion; Steroids; Systematic Review; Therapeutics.

\section{INTRODUCTION}

Postherpetic neuralgia (PHN), a persistent neuropathic pain that develops after acute herpes zoster (HZ), is the most frequent chronic complication of $\mathrm{HZ}$ [1]. HZ is caused by the reactivation of the varicella zoster virus (VZV), a highly contagious double-stranded DNA virus that causes chickenpox. The reactivation of VZV is associated with (c) This is an open-access article distributed under the terms of the Creative Commons Attribution Non-Commercial License (http://creativecommons.org/licenses/by-nc/4.0/), which permits unrestricted non-commercial use, distribution, and reproduction in any medium, provided the original work is properly cited.

(c) The Korean Pain Society, 2021
Author contributions: Junhyeok Kim: Methodology; Min Kyoung Kim: Writing/manuscript preparation; Geun Joo Choi: Investigation; Hwa Yong Shin: Data curation; Beom Gyu Kim: Writing/manuscript preparation; Hyun Kang: Writing/manuscript preparation. 
age-related decrease in cellular immunity to VZV and impaired cellular immune function [2]. The estimated overall incidence of $\mathrm{HZ}$ is 3.4-4.82/1,000 person-year and increases up to $11 / 1,000$ person-year in patients aged 80 years and older. The lifetime risk ranges between $25 \%$ and $30 \%$ but is up to $50 \%$ for individuals older than 80 years [3]. HZ has recently been linked to an increased risk of cerebrovascular and cardiac events in the days following an acute infection [4], and is considered as a major public health problem because of its increasing incidence and because it is common in the aging population [1].

The risk of developing PHN in individuals with $\mathrm{HZ}$ is between $5 \%$ and $30 \%$. Prodromal pain, older age, greater acute pain, a more widespread rash, and ophthalmic involvement are major risk factors for PHN [5]. The pain characteristics of PHN have been described as burning, aching, throbbing, stabbing, or shooting, and it may be continuous or intermittent. Allodynia, hyperalgesia, and dysesthesia were also observed. The pain severity varies from mild to excruciating; in some patients, the pain is intractable and leads to depression, fatigue, and sleep disturbance $[6,7]$. This severe chronic pain also leads to various socioeconomic consequences, including decreased socialization, daily activities, and quality of life $[8,9]$. Therefore, the prevention of PHN is a major objective in the treatment of $\mathrm{HZ}$, along with treatment of the acute viral infection and acute pain. As the pathophysiological mechanisms of PHN are complex, various preventive strategies, including antiviral agents, vaccination, corticosteroids, antidepressants, anticonvulsants, and nerve blocks have been introduced. However, it remains unclear which strategies are more effective in preventing PHN.

Recently, a few systematic reviews and meta-analyses have investigated the preventive effects of various strategies [10-13]. However, each of systematic reviews and meta-analyses was only performed using a pair-wise approach; thus, only two strategies were compared. No previous network meta-analysis (NMA) has compared the effectiveness of all available strategies to prevent PHN. Furthermore, these meta-analyses only included studies conducted before 2014 .

NMA, an extension of traditional pair-wise meta-analysis, is a research method that can compare and analyze comparative studies simultaneously by combining direct and indirect evidence in the network of the existing randomized controlled trials (RCT); it provides a relative efficacy and a hierachy of various treatments based on the corresponding surface under the cumulative ranking curve (SUCRA) value [14].

Thus, we reviewed all articles that investigated the effects of various strategies employed to prevent PHN and quantified the rank order of the efficacy of various strate- gies for preventing PHN using NMA.

\section{MATERIALS AND METHODS}

\section{Protocol and registration}

We developed the protocol for this systematic review and NMA in accordance with the Preferred Reporting Items for Systematic Review and Meta-analysis (PRISMA) statement [15]. The review protocol was registered at the International Prospective Register of Systematic Reviews (registration number: CRD42021225666; accessible at https://www.crd.york.ac.uk/prospero/display_record. php?RecordID=225666) on October 1, 2020.

This systematic review and NMA of pharmacological and non-pharmacological strategies for preventing PHN was performed according to the protocol recommended by the Cochrane Collaboration [16] and reported according to the PRISMA extension for NMA guidelines [17].

\section{Search strategy}

We searched MEDLINE, EMBASE, Cochrane Central Register of Controlled Trials (CENTRAL), and Google Scholar from database establishment (MEDLINE; 1,946, EMBASE; $1,966)$ to December 2020 using search terms related to the pharmacological and non-pharmacological strategies for preventing PHN and updated it in June 2021. The search terms used for MEDLINE and EMBASE are presented in the Appendix. Two investigators (JHK and GJC) screened the titles and abstracts of the retrieved articles. The reference lists were imported to Endnote software 9.1 (Clarivate, London, UK), and duplicate articles were removed. Additional relevant articles were identified by scanning the reference lists of the articles obtained from the original search.

\section{Inclusion criteria and exclusion criteria}

We only included prospective RCTs that compared two or more pharmacological and non-pharmacological strategies for preventing PHN.

The PICO-SD information is as follows:

1. Patients (P): all patients with acute $\mathrm{HZ}$

2. Intervention (I): pharmacological and non-pharmacological strategies employed to prevent PHN

3. Comparison (C): other pharmacological and nonpharmacological strategies employed to prevent PHN, placebo, or no treatment

4. Outcome measurements $(\mathrm{O})$ : The primary outcome 
of this NMA was the incidence of PHN at 3 months after acute HZ. The secondary outcomes of this NMA were the incidence of PHN at 1 and 6 months after acute $\mathrm{HZ}$, and severity of pain measured at 1,3 , and 6 months after acute $\mathrm{HZ}$ was also analyzed.

When the data for 1,3 , and 6 months was not presented, we included the data from the nearest time point, if possible.

5. Study design (SD): RCTs

The following types of studies were excluded:

1. Review articles, case reports, case series, letters to the editor, commentaries, proceedings, laboratory science studies, and all other non-relevant studies

2. Studies that failed to report the outcomes of interest

No language or date restrictions were applied in our study.

\section{Study selection}

Two investigators (JHK and GJC) independently screened the titles and abstracts of the searched studies to identify trials that met the inclusion criteria outlined above. For articles determined to be eligible for the analysis based on their titles and/or abstracts, the full paper was retrieved. Potentially relevant studies chosen by at least one investigator were retrieved, and the full text was evaluated. Fulltext articles were assessed separately by two investigators (JHK and GJC), and any disagreements were resolved through discussion. In cases where agreement could not be reached, the dispute was resolved with the help of a third investigator (HK). To minimize data duplication because of multiple reports, articles from the same author, organization, or country were compared.

The degree of agreement for study selection between the two independent investigators was computed using kappa statistics to measure the difference between the observed and expected agreements, that is, whether they were random or by chance. Kappa values were interpreted as follows: 1) less than 0: less than chance agreement; 2) 0.01-0.20: slight agreement; 3) 0.21-0.40: fair agreement; 4) 0.41-0.60: moderate agreement; 5) 0.61-0.80: substantial agreement; and 6) 0.8-0.99: almost perfect agreement [18].

\section{Data extraction}

Using a standardized extraction form, the following data were extracted independently by two investigators (JHK and MKK): 1) title; 2) name of the first author; 3) name of the journal; 4) year of publication; 5) study design; 6) type of pharmacological and non-pharmacological strategies; 7) dose of pharmacological agents; 8) country; 9) risk of bias; 10) inclusion criteria; 11) exclusion criteria; 12) age; 13) sex; 14) number of subjects; 15) incidence of PHN at 1, 3, and 6 months after acute HZ; and 16) pain score measured at 1,3 , and 6 months after acute HZ.

If the information were inadequate, attempts were made to contact the study authors, and additional information was requested. If unsuccessful, missing information was calculated from the available data, if possible, or was extracted from the figure using the open source software Plot Digitizer (version 2.6.8; http://plotdigitizer.sourceforge. net).

The reference lists were divided into two portions. Two investigators (HYS and MKK) completed the data extraction, one for each half of the reference list. Data extraction forms were cross-checked to verify the accuracy and consistency of the extracted data.

\section{Risk of bias}

Risk of bias was assessed using the revised Cochrane risk of bias tool for randomized trials (RoB 2.0 version) (August 22,2019 ) by two independent authors (BGK and HK) [19]. The RoB 2.0 is structured into five domains: D1) bias arising from the randomization process, D2) bias due to deviations from the intended interventions, D3) bias due to missing outcome data, D4) bias in outcome measurements, D5) bias in the selection of reported results. The overall risk of bias was evaluated. It was judged as low risk when the risk of bias in all domains was low, high when the risk of bias in at least one domain was high or the risk of bias in multiple domains was of some concern, and some concern if the overall judgement neither low nor high.

\section{Statistical analyses}

Ad-hoc tables were created to summarize the data from the included studies by showing their key characteristics and any important questions related to the review objectives. After extracting the data, the investigators determined the feasibility of the meta-analysis.

A multiple treatment comparison NMA is a metaanalysis generalization method that includes both direct and indirect comparisons of treatments. Both frequentist and Bayesian random-effects NMAs were conducted. A frequentist random-effects NMA was performed using the STATA software (version 15; StataCorp LP, College Station, TX) based on mvmeta with NMA graphical tools developed by Chaimani et al. [20].

Before conducting the NMA, we evaluated the transitivity assumption by examining the comparability of demographic data (age and sex), type of strategies for preventing PHN, and the risk of bias (low vs. removing low risk of bias 
from the overall risk of bias) as potential treatment-effect modifiers across comparisons.

A network plot linking the included pharmacological and non-pharmacological strategies for preventing PHN and their combination with other pharmacological and non-pharmacological strategies was constructed to indicate the types of pharmacological and non-pharmacological strategies, number of patients on different strategies, and the level of pair-wise comparisons. The nodes show the pharmacological and non-pharmacological strategies compared, and the edges show the available direct comparisons among the pharmacological and non-pharmacological strategies. The nodes and edges are weighed on the basis of the number of patients and the inverse of standard error of the effect.

We evaluated the consistency assumption within the entire network using the design-by-treatment interaction model. We also evaluated each closed loop in the network to evaluate the local inconsistencies between the direct and indirect effect estimates for the same comparison. For each loop, we estimated the inconsistency factor (IF) as the absolute difference between the direct and indirect estimates and the corresponding 95\% confidence interval (CI) for each paired comparison in the loop [21]. When the IF value with $95 \% \mathrm{CI}$ started at 0 , it indicated that the direct and indirect evidence were consistent.

In the predictive interval (PrI) plot, the mean summary effects with CI were presented together with their PrIs to facilitate interpretation of the results, considering the magnitude of heterogeneity. PrIs provide an interval that is expected to encompass the estimate of a future study. A rankogram and cumulative ranking curve were drawn for each pharmacological and non-pharmacological strategy. Rankograms are the probabilities for treatments to assume a possible rank. We used the SUCRA values to present the hierarchy of pharmacological and non-pharmacological strategies for preventing PHN. SUCRA is a relative ranking measure that accounts for the uncertainty in the treatment order; it accounts for both the location and variance of all relative treatment effects. A higher SUCRA value indicates that the individual interventions have more positive results [22]. A comparison-adjusted funnel plot was used to assess the presence of small-study effects [23].

To test the robustness of the results from frequentist random NMA, we also conducted a Bayesian NMA using the fixed and random-effects models as well as Markov Chain Monte Carlo (MCMC) methods with the R statistical package gemtc [24]. Due to a lack of understanding on PHN, we used uninformative prior distributions automatically provided by the gemtc package. MCMC simulations were run using four chains with different initial values for an inferential 100,000 iterations after 50,000 burn-ins and thin- ning of 100. The convergence of fixed and random models derived from MCMC simulations was assessed using trace and density plots, and Gelman-Rubin-Brooks methods with a potential scale reduction factor (PSRF) of up to 1 . And the comparing of the fit for fixed and random models was assessed using Dbar (posterior mean of the deviance), PD (adequate number of parameters), and DIC (deviance information criterion, the sum of Dbar and PD) statistics. We also calculated the SUCRA values from the Bayesian model and compared them with those in the frequentist model. We also performed a meta-regression analysis considering the potential influences of the risk of bias (low risk vs. some concern or high risk), sex, and mean age on each outcome.

\section{Quality of evidence}

The evidence grade was determined using the Grading of Recommendations, Assessment, Development, and Evaluation (GRADE) system, which involves a sequential assessment of the evidence quality, an assessment of the risk-benefit balance, and a subsequent judgment on the strength of the recommendations [25].

\section{RESULTS}

\section{Study selection}

A total of 1,090 studies were found after searching MEDLINE, EMBASE, CENTRAL, and Google Scholar, with an additional 77 studies discovered after conducting a manual search, such as looking at references from included studies/reviews, and additional searching of related/cited articles in PubMed and Google Scholar. A total of 1,070 studies were retained after removing duplicates. After reviewing the titles and abstracts, 972 studies were excluded. In the first stage of the study selection process, the kappa value between two investigators was 0.764 .

The remaining 98 studies were thoroughly examined, and 59 were eliminated for the following reasons: Metaanalysis $[10,13,26-33]$, were irrelevant to outcomes of the study [34-55], unavailable outcomes [56-67], non-original data [68-71], grouping [72-77], and were nonrandomized studies [78-82]. As a result, 39 studies met the criteria for inclusion in this systematic review and meta-analysis (Fig. 1). In the second stage of the study selection process, the kappa value between two investigators was 0.834 .

\section{Study characteristics}

Table 1 summarizes the characteristics of the 39 stud- 


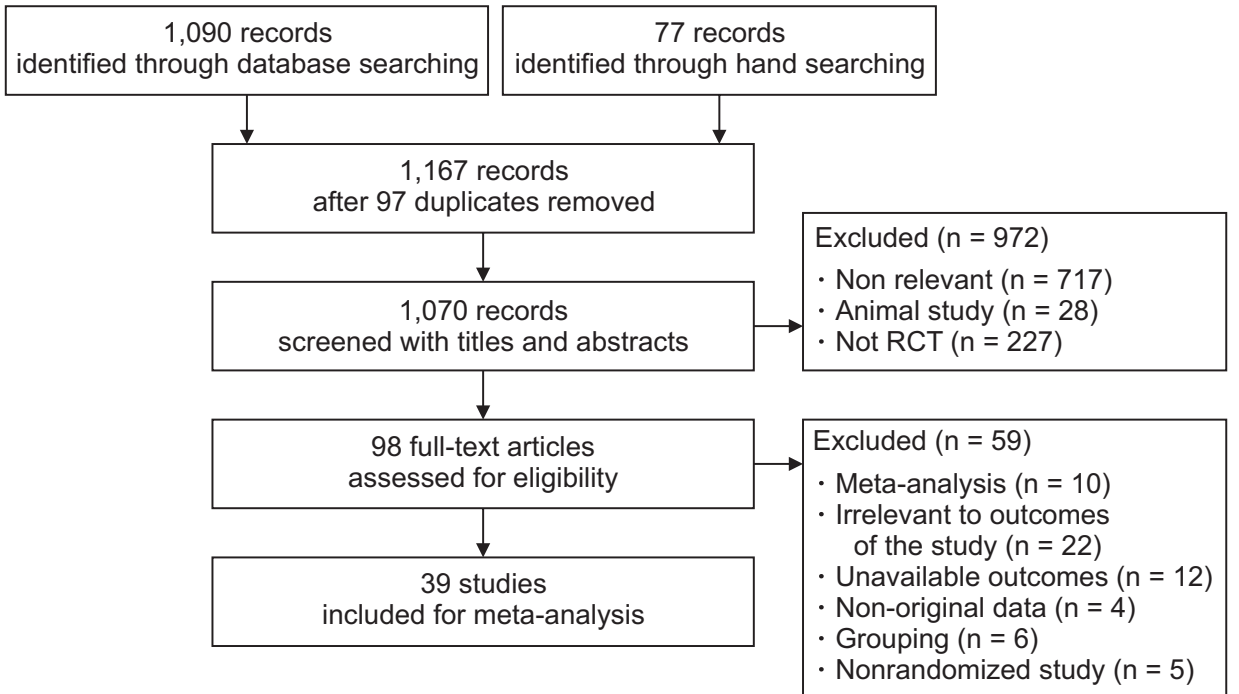

Fig. 1. Preferred Reporting Items for Systematic Review and Meta-analysis (PRISMA) flowchart of included and excluded trials. RCT: randomized controlled trial. ies that met the inclusion criteria. The pharmacological and non-pharmacological strategies used to prevent PHN consisted of antiviral agents (AV) [83-109]; a combination of antiviral agents and antiepileptics (AV + AE) [93,98101,104,107-109]; electric nerve stimulation (ENS) [93,103]; a combination of antiviral agents and electric nerve stimulation (AV-ENS) [103]; a combination of antiviral agents, antiepileptics, and paravertebral block using local anesthetics and steroids (AV + AE-pv) [97,102]; a combination of antiviral agents and epidural block using local anesthetics and steroids (AV-eLS) [96]; a combination of antiviral agents and steroids $(\mathrm{AV}+\mathrm{SR})[88,110,111]$; continuous epidural block using local anesthetics, steroids and epinepherine (EPI-LSE) [110]; steroids (SR) [92,112-115]; antiepileptics (AE) [92,93,115]; a combination of antiviral agents and intracutaneous injection using local anesthetics and steroids (AV + iLS) [105]; a combination of antiviral agents and subcutaneous injection using local anesthetics and steroids (AV + sLS) [106]; varicella zoster vaccine immunoglobulin (VZVIG) [116]; adrenocorticotropic hormone (ACTH) [112]; a combination of antivial agents and intracutaneous injection using methylene blue and local anesthetics (AV-iLM) [117]; a combination of antiviral agents and intracutaneous injection using local anesthetics (AViL) [105]; a combination of antiviral agents and continuous \& intermittent epidural block using local anesthetics (AVecL) [118]; a combination of antiviral agents and intermittent epidural block using local anesthetics (AV-eiL) [118]; radiotherapy (RTx) [92]; a combination of antiviral agents, antiepileptics and stellate ganglion block using local anesthetics and steroids (AV + AE-sgLS) [101]; a combination of antiviral agents and stellate ganglion block using local anesthetics (AV-sgL) [95]; a combination of antiviral agents, antiepileptics, and intracutaneous injection using local anesthetics and steroids (AV + AE-iLS) [107]; a combination of antiviral agents, antiepileptics and cervical nerve root block using local anesthetics, steroids, and cobalamide (AV + AE-cLSC) [109]; stellate ganglion block using local anesthetics (sgL) [119]; and pulsed radiofrequency on the Gasserian ganglion (pRF) [120].

\section{Risk of bias assessment}

The risk of bias assessment is described in Table 2.

\section{Synthesis of results}

With regard to the outcomes of each specific datum, we generated a network plot (Fig. 2), inconsistency plot (Fig. 3), a predictive interval plot compared with the control (Fig. 4), expected mean ranking and the SUCRA values for each strategy (Fig. 5), the comparison-adjusted funnel plot (Fig. 6), and the SUCRA values from the Bayesian model compared with the SUCRA values from the frequentist model (Fig. 7). Figs. 2-7 present a summary of the results (A, B, C, $\mathrm{D}, \mathrm{E}$, and $\mathrm{F}$ in each figure correspond to the incidence of PHN at 3 months, incidence of PHN at 1 month, incidence of PHN at 6 months, pain score at 3 months, pain score at 1 month, and pain score at 6 months, respectively).

\section{Incidence of PHN at 3 months after acute $\mathrm{HZ}$}

A total of 27 studies (3,136 patients) measured the incidence of PHN at 3 months after acute HZ. Of these, one study was separated from the loops [117]. NMA was performed excluding the abovementioned study. Thus, only 26 studies (3,072 patients) were analyzed. The network plot of all eligible comparisons for this endpoint is shown in Fig. 2A. Although all 17 management modalities (nodes) were connected to the network, two comparisons (AV and 
Table 1. Characteristics of the trials included in the systematic review and network meta-analysis

\begin{tabular}{|c|c|c|c|c|c|c|c|c|c|c|c|}
\hline \multirow[b]{2}{*}{ Author, year } & \multirow[b]{2}{*}{ Management } & \multirow[b]{2}{*}{$\begin{array}{c}\text { Number } \\
\text { of } \\
\text { patients }\end{array}$} & \multirow[b]{2}{*}{ Age, yr } & \multirow[b]{2}{*}{ Sex, M/F } & \multirow[b]{2}{*}{$\begin{array}{c}\text { Pain } \\
\text { assessment } \\
\text { tool }\end{array}$} & \multicolumn{6}{|c|}{ Outcomes of interest } \\
\hline & & & & & & $\begin{array}{l}\text { Incidence } \\
\text { of PHN at } \\
1 \text { month }\end{array}$ & $\begin{array}{l}\text { Incidence } \\
\text { of PHN at } \\
3 \text { months }\end{array}$ & $\begin{array}{l}\text { Incidence } \\
\text { of PHN at } \\
6 \text { months }\end{array}$ & $\begin{array}{c}\text { Mean } \\
\text { pain } \\
\text { score at } \\
1 \text { month }\end{array}$ & $\begin{array}{c}\text { Mean } \\
\text { pain } \\
\text { score at } \\
3 \text { months }\end{array}$ & $\begin{array}{c}\text { Mean } \\
\text { pain } \\
\text { score at } \\
6 \text { months }\end{array}$ \\
\hline \multirow{2}{*}{$\begin{array}{l}\text { Bulilete et al., } 2019 \\
\text { [108] }\end{array}$} & Valaciclovir + gabapentin & 50 & 65.1 & $20 / 28$ & 10-point VAS & - & - & & - & - & \\
\hline & Valaciclovir + placebo & 48 & 66.0 & $18 / 30$ & & & & & & & \\
\hline \multirow[t]{2}{*}{ Lee et al., 2016 [104] } & Valaciclovir + gabapentin & 60 & 62.58 & $18 / 42$ & 10-point Likert & - & $\bullet$ & & • & - & \\
\hline & Valaciclovir & 60 & 61.83 & $25 / 35$ & scale & & & & & & \\
\hline \multirow{4}{*}{$\begin{array}{l}\text { Stepanović et al., } 2015 \\
\text { [103] }\end{array}$} & TENS & 36 & 57.3 & $14 / 22$ & VAS & • & $\bullet$ & - & & & \\
\hline & Symptomatic care & 38 & 59.9 & $16 / 22$ & & & & & & & \\
\hline & Antiviral agent & 71 & 70.6 & $32 / 39$ & & & & & & & \\
\hline & Antiviral + TENS & 77 & 65.6 & $27 / 50$ & & & & & & & \\
\hline \multirow{2}{*}{$\begin{array}{l}\text { Makharita et al., } 2015 \\
\text { [102] }\end{array}$} & Acyclovir + pregabalin + saline (paravertebral) & 70 & 56.2 & $31 / 37$ & VAS & & • & • & • & • & • \\
\hline & $\begin{array}{l}\text { Acyclovir }+ \text { pregabalin }+ \text { bupivacaine }+ \text { dexamethasone } \\
\quad(\text { paravertebral })\end{array}$ & 73 & 56.8 & $34 / 36$ & & & & & & & \\
\hline \multirow{2}{*}{$\begin{array}{l}\text { Makharita et al., } 2012 \\
\text { [101] }\end{array}$} & Antiviral + pregabalin + saline (SGB) & 30 & 59.6 & $14 / 16$ & VAS & & • & • & - & • & • \\
\hline & $\begin{array}{l}\text { Antiviral + pregabalin + bupivacaine + dexamethasone } \\
\text { (SGB) }\end{array}$ & 30 & 60.6 & $13 / 18$ & & & & & & & \\
\hline \multirow{2}{*}{$\begin{array}{l}\text { Krcevski Skvarc and } \\
\text { Kamenik, } 2010 \text { [98] }\end{array}$} & Antiviral + placebo & 15 & 63 & $4 / 11$ & 10 point Likert & & • & • & • & & \\
\hline & Antiviral + pregabalin & 14 & 67 & $6 / 8$ & scale & & & & & & \\
\hline \multirow[t]{2}{*}{ Ji et al., 2009 [97] } & Acyclovir & 64 & 68 & $28 / 36$ & VAS & - & • & • & • & - & • \\
\hline & $\begin{array}{l}\text { Acyclovir }+ \text { bupivacaine }+ \text { methylprednisolone } \\
\text { (paravertebral) }\end{array}$ & 68 & 66 & $30 / 38$ & & & & & & & \\
\hline \multirow{2}{*}{$\begin{array}{l}\text { van Wijck et al., } 2006 \\
\text { [96] }\end{array}$} & Acyclovir & 297 & 66 & $116 / 181$ & VAS & - & & & - & • & • \\
\hline & Acyclovir + bupivacaine + methylprednisolone (epidural) & 301 & 66 & $118 / 183$ & & & & & & & \\
\hline \multirow{2}{*}{$\begin{array}{l}\text { Pasqualucci et al., } 2000 \\
\text { [110] }\end{array}$} & Acyclovir + prednisolone & 279 & 66.9 & $125 / 154$ & VAS & - & $\bullet$ & • & • & • & • \\
\hline & $\begin{array}{l}\text { Bupivacaine + epinephrine + methylprednisolone via } \\
\text { epidural catheter }\end{array}$ & 290 & 68.7 & $131 / 159$ & & & & & & & \\
\hline Ahmed et al., 1998 [94] & Famciclovir & 25 & 53 & $11 / 14$ & VAS $100 \mathrm{~mm}$ & & $\bullet$ & - & - & - & - \\
\hline & PENS & 25 & 56 & $12 / 13$ & & & & & & & \\
\hline Bowsher, 1997 [93] & Acyclovir + amitriptyline & 9 & 71.3 & $14 / 24$ & NR & $\bullet$ & $\bullet$ & - & & & \\
\hline & Amitriptyline & 29 & & & & & & & & & \\
\hline & Acyclovir + placebo & 17 & 72.7 & $14 / 20$ & & & & & & & \\
\hline & Placebo & 17 & & & & & & & & & \\
\hline Harding and Porter, & Acyclovir & 24 & 62.1 & $6 / 17$ & VAS $100 \mathrm{~mm}$ & & & & - & - & - \\
\hline $1991[114]$ & Placebo & 22 & 70.6 & $9 / 19$ & & & & & & & \\
\hline Benoldi et al., 1991 [92] & Prednisolone & 9 & 68.5 & $4 / 5$ & NR & & - & - & & & \\
\hline & $\mathrm{RTX}$ & 9 & 67.2 & $3 / 6$ & & & & & & & \\
\hline & Acyclovir & 9 & 67.1 & $6 / 3$ & & & & & & & \\
\hline & Carbamazepine & 9 & 63 & $3 / 6$ & & & & & & & \\
\hline Esmann et al., 1987 & Acyclovir + prednisolone & 41 & 72.8 & $17 / 24$ & Slight, moderate, & - & - & & & & \\
\hline$[88]$ & Acyclovir + placebo & 37 & 71.4 & $8 / 29$ & $\begin{array}{l}\text { sever, attacks } \\
\text { per day, highest } \\
\text { grading }\end{array}$ & & & & & & \\
\hline Cobo et al., 1986 [87] & Acyclovir & 36 & NR & $13 / 23$ & None, mild, mod- & & • & & & & \\
\hline & Placebo & 35 & & $21 / 14$ & erate, severe & & & & & & \\
\hline Balfour et al., 1983 [85] & Acyclovir & 52 & NR & $29 / 23$ & NR & - & • & & & & \\
\hline & Placebo & 42 & & $25 / 17$ & & & & & & & \\
\hline Esmann et al., 1982 & Acyclovir & 27 & 65.7 & $10 / 17$ & NR & - & • & & & & \\
\hline$[84]$ & Placebo & 29 & 68.6 & $10 / 19$ & & & & & & & \\
\hline Bean et al., 1982 [83] & Acyclovir & 19 & 53.2 & $6 / 13$ & NR & - & • & & & & \\
\hline & Placebo & 10 & 50.5 & $7 / 3$ & & & & & & & \\
\hline Keczkes and Basheer, & Prednisolone & 20 & 66.4 & $14 / 6$ & NR & • & & & & & \\
\hline $1980[115]$ & Carbamazepine & 20 & 68.5 & $14 / 6$ & & & & & & & \\
\hline Cui et al., 2018 [107] & $\begin{array}{l}\text { Acyclovir }+ \text { pregabalin }+ \text { ropivacaine }+ \text { methylpredniso- } \\
\text { lone (intracutaneous) }\end{array}$ & 51 & 61.7 & $21 / 28$ & VAS & • & • & • & • & • & • \\
\hline & Acyclovir + pregabalin + saline (intracutaneous) & 51 & 61.8 & $20 / 28$ & & & & & & & \\
\hline Cui et al., 2017 [105] & $\begin{array}{l}\text { Acyclovir + ropivacaine + methylprednisolone (intracuta- } \\
\text { neous) }\end{array}$ & 48 & 63.7 & $21 / 26$ & VAS & • & • & • & • & • & • \\
\hline & Acyclovir & 48 & 63.0 & $19 / 27$ & & & & & & & \\
\hline Ni et al., 2017 [106] & Acyclovir + triamcinolone + lidocaine (subcutaneous) & 50 & 63.84 & $23 / 27$ & NRS & • & • & • & & & \\
\hline & Acyclovir & 50 & 65.86 & $24 / 26$ & & & & & & & \\
\hline Zheng et al., 2019 [109] & Famciclovir + pregabalin + placebo (cervical root block) & 70 & 63.41 & $31 / 39$ & 11 point scale & & • & • & • & • & • \\
\hline & $\begin{array}{l}\text { Famciclovir + pregabalin + lidocaine }+ \text { triamcinolone }+ \\
\text { cobalamine (cervical root block) }\end{array}$ & 70 & 65.84 & $29 / 41$ & & & & & & & \\
\hline
\end{tabular}


Table 1. Continued

\begin{tabular}{|c|c|c|c|c|c|c|c|c|c|c|c|}
\hline \multirow[b]{2}{*}{ Author, year } & \multirow[b]{2}{*}{ Management } & \multirow[b]{2}{*}{$\begin{array}{l}\text { Number } \\
\text { of } \\
\text { patients }\end{array}$} & \multirow[b]{2}{*}{ Age, yr } & \multirow[b]{2}{*}{ Sex, M/F } & \multirow[b]{2}{*}{$\begin{array}{c}\text { Pain } \\
\text { assessment } \\
\text { tool }\end{array}$} & \multicolumn{6}{|c|}{ Outcomes of interest } \\
\hline & & & & & & $\begin{array}{l}\text { Incidence } \\
\text { of PHN at } \\
1 \text { month }\end{array}$ & $\begin{array}{l}\text { Incidence } \\
\text { of PHN at } \\
3 \text { months }\end{array}$ & $\begin{array}{l}\text { Incidence } \\
\text { of PHN at } \\
6 \text { months }\end{array}$ & $\begin{array}{l}\text { Mean } \\
\text { pain } \\
\text { score at } \\
1 \text { month } 3\end{array}$ & $\begin{array}{c}\text { Mean } \\
\text { pain } \\
\text { score at } \\
3 \text { months }\end{array}$ & $\begin{array}{c}\text { Mean } \\
\text { pain } \\
\text { score at } \\
6 \text { months }\end{array}$ \\
\hline \multirow[t]{2}{*}{ Hwang et al., 1999 [79] } & $\begin{array}{l}\text { Acyclovir + bupivacaine + methylprednisolone (continu- } \\
\text { ous epidural infusion) }\end{array}$ & 40 & 60.8 & $18 / 22$ & VRS 0-100 & & - & & & & \\
\hline & Acyclovir & 35 & 56.1 & $9 / 26$ & & & & & & & \\
\hline \multirow[t]{2}{*}{ Wan et al., 2019 [120] } & Sham & 48 & 64.87 & $20 / 28$ & VAS & & & & - & - & - \\
\hline & PRF on gasserian ganglion & 48 & 66.01 & $23 / 25$ & & & & & & & \\
\hline \multirow{2}{*}{$\begin{array}{l}\text { Hügler et al., } 2002 \\
\text { [116] }\end{array}$} & Human albumin (placebo) & 20 & 67.65 & NR & VAS & - & & & & & \\
\hline & VZVIG & 20 & 71.6 & & & & & & & & \\
\hline \multirow[t]{2}{*}{ Cui et al., 2016 [117] } & Valacyclovir + methylene blue + lidocaine (intradermal) & 32 & 69.5 & $13 / 19$ & VAS $100 \mathrm{~mm}$ & - & - & & - & & \\
\hline & Valacyclovir + lidocaine (intradermal) & 32 & 72.5 & $11 / 21$ & & & & & & & \\
\hline \multirow[t]{2}{*}{ Payne et al., 1989 [91] } & Placebo & 17 & 70 & $10 / 7$ & NR & - & - & - & & & \\
\hline & Isoprinosine & 21 & 70 & 10/11 & & & & & & & \\
\hline \multirow[t]{4}{*}{ Wood et al., 1994 [111] } & Acyclovir 7 days & 101 & 58 & $39 / 62$ & $0-5 ;$ non- & - & & & & & \\
\hline & Acyclovir 7 days + prednisoone 21 days & 99 & 59 & $37 / 62$ & noticeable to & & & & & & \\
\hline & Acyclovir 21 days & 101 & 59 & $38 / 63$ & excruciating & & & & & & \\
\hline & Acyclovir 21 days + prednisolone 21 days & 99 & 60 & $39 / 60$ & & & & & & & \\
\hline \multirow[t]{2}{*}{ McGill et al., 1983 [86] } & Placebo & 20 & 68.8 & $7 / 13$ & $0-3$ & & - & & & & \\
\hline & Acyclovir & 17 & 70.4 & $3 / 14$ & & & & & & & \\
\hline \multirow{2}{*}{$\begin{array}{l}\text { Wassilew et al., } 1987 \\
\text { [89] }\end{array}$} & Acyclovir & 29 & 62.5 & $9 / 20$ & 0-5 & - & - & - & & & \\
\hline & Placebo & 31 & 63.4 & $6 / 25$ & & & & & & & \\
\hline \multirow{2}{*}{$\begin{array}{l}\text { Mandal et al., } 1988 \\
\text { [90] }\end{array}$} & Acyclovir & 26 & 67.4 & $11 / 15$ & $0-4$ & - & & & & & \\
\hline & Placebo & 30 & 68.4 & $9 / 21$ & & & & & & & \\
\hline \multirow[t]{2}{*}{ Lee et al., 1999 [95] } & Acyclovir + mepivacaine (stellate ganglion block) & 10 & 65.0 & $3 / 7$ & VAS 0-100 mm & & & & - & & • \\
\hline & Acyclovir & 10 & 67.2 & $5 / 5$ & & & & & & & \\
\hline \multirow{2}{*}{$\begin{array}{l}\text { Harding et al., } 1986 \\
\text { [119] }\end{array}$} & $1 \%$ lignocaine \& $0.5 \%$ marcaine (stellate ganglion block) & NR & 71.5 & NR & VAS $0-100 \mathrm{~mm}$ & & & & - & - & \\
\hline & Placebo & & 72.2 & & & & & & & & \\
\hline \multirow{2}{*}{$\begin{array}{l}\text { Kanodia and Singhal, } \\
2011 \text { [99] }\end{array}$} & Acyclovir + pregabalin & 23 & 46 & $19 / 4$ & VAS $100 \mathrm{~mm}$ & & & & - & & \\
\hline & Acyclovir + placebo & 22 & 47 & $17 / 5$ & & & & & & & \\
\hline \multirow{4}{*}{$\begin{array}{l}\text { Kanodia et al., } 2012 \\
\text { [100] }\end{array}$} & Acyclovir + gabapentin 300 mg/day & 15 & 64 & $11 / 4$ & VAS $100 \mathrm{~mm}$ & & & & - & & \\
\hline & Acyclovir + gabapentin 600 mg/day & 14 & 65 & $9 / 5$ & & & & & & & \\
\hline & Acyclovir + gabapentin 900 mg/day & 13 & 65 & $10 / 3$ & & & & & & & \\
\hline & Placebo & 14 & 63 & $11 / 3$ & & & & & & & \\
\hline \multirow[t]{2}{*}{$\begin{array}{l}\text { Manabe et al., } 2004 \\
\text { [118] }\end{array}$} & $\begin{array}{l}\text { Acyclovir + bupivacaine (continous epidural infusion, } \\
\text { intermittent epidural bolus) }\end{array}$ & 29 & 67 & $9 / 20$ & VAS & • & & & & & \\
\hline & $\begin{array}{l}\text { Acyclovir }+ \text { normal saline (continous epidural infusion) + } \\
\text { bupivacaine (intermittent epidural bolus) }\end{array}$ & 27 & 65 & $13 / 14$ & & & & & & & \\
\hline \multirow{3}{*}{$\begin{array}{l}\text { Clemmensen and } \\
\text { Andersen, } 1984 \\
\text { [112] }\end{array}$} & ACTH & 17 & 55 & $10 / 7$ & $0-4$ & - & & & & & \\
\hline & Prednisolone & 19 & 56 & $13 / 6$ & & & & & & & \\
\hline & Placebo & 19 & 56 & $10 / 9$ & & & & & & & \\
\hline \multirow{2}{*}{$\begin{array}{l}\text { Eaglstein et al., } 1970 \\
\text { [113] }\end{array}$} & Triamcinolone & 15 & NR & NR & NR & & • & & & & \\
\hline & Placebo & 19 & & & & & & & & & \\
\hline
\end{tabular}

control [CTR]) were more directly compared with the other 15 nodes. No network inconsistency was observed $\left[\chi^{2}(7)=\right.$ 8.94, $P=0.257$ ].

Ten closed loops were identified in the network after compariing the incidence of $\mathrm{PHN}$ at 3 months after acute $\mathrm{HZ}$, but four loops (CTR/AV + AE/AE [93], CTR/ENS/AVENS [103], AV/SR/RTx [92], and SR/RTx/AE [92]) were formed only by multi-arm trials. Of the six closed loops, inconsistencies were observed in 1/10/12 (CTR/SR/AE) (Fig. 3A). EPI-LSE showed a lower incidence of PHN at 3 months after acute HZ than CTR in terms of $95 \% \mathrm{CI}$ and PrI; moreover, AV + iLS and AV + AE-pv showed a lower incidence of PHN at 3 months after acute HZ than CTR, but only in terms of 95\% CI (Fig. 4A, Supplementary Fig. 1A,
Supplementary Table 1A). The insignificances in the $95 \%$ PrIs suggests that any future RCTs could change the significance of the efficacy of these comparisons. The rankograms and cumulative ranking curve showed that EPI-LSE followed by AV + sLS and AV + iLS had the lowest incidence of PHN at 3 months after acute HZ (Supplementary Figs. 1B, 1C). The expected mean rankings and SUCRA values of each intervention are presented in Fig. 5A. According to the SUCRA value, the incidence of PHN at 3 months after acute HZ was lower in the order of the EPI-LSE (97.0\%), followed by AV + sLS (79.5\%), AV + iLS (79.2\%), and AV + AE$\operatorname{sgLS}(76.7 \%)$.

The comparison-adjusted funnel plots showed that the funnel plots were symmetrical around the zero line, which 
Table 2. Risk of bias assessment

\begin{tabular}{|c|c|c|c|c|c|c|c|c|c|c|c|}
\hline \multirow{2}{*}{$\begin{array}{c}\text { Study, year } \\
\text { Bulilete et al., } \\
2019 \text { [108] }\end{array}$} & \multicolumn{2}{|c|}{ Randomization process } & \multicolumn{2}{|c|}{ Intended interventions } & \multicolumn{2}{|c|}{ Missing outcome data } & \multicolumn{2}{|c|}{$\begin{array}{l}\text { Measurement of the } \\
\text { outcome }\end{array}$} & \multicolumn{2}{|c|}{$\begin{array}{l}\text { Selection of the reported } \\
\text { result }\end{array}$} & \multirow{2}{*}{$\begin{array}{l}\begin{array}{c}\text { Overall } \\
\text { result }\end{array} \\
\text { Some } \\
\text { concern }\end{array}$} \\
\hline & $\begin{array}{l}\text { Some } \\
\text { concern }\end{array}$ & $\begin{array}{l}\text { No statement for allo- } \\
\text { cation concealment }\end{array}$ & Low risk & Both blinded & Low risk & $\begin{array}{l}\text { 1/98 dropped, } \\
\text { unrelated to } \\
\text { the outcome }\end{array}$ & Low risk & Blinded & Low risk & $\begin{array}{l}\text { Predefined } \\
\text { outcomes }\end{array}$ & \\
\hline $\begin{array}{l}\text { Lee et al., } 2016 \\
\text { [104] }\end{array}$ & $\begin{array}{l}\text { Some } \\
\text { concern }\end{array}$ & $\begin{array}{l}\text { No statement for allo- } \\
\text { cation concealment }\end{array}$ & $\begin{array}{l}\text { Some } \\
\text { concern }\end{array}$ & $\begin{array}{l}\text { No specific } \\
\text { information }\end{array}$ & $\begin{array}{l}\text { Some } \\
\text { concern }\end{array}$ & $\begin{array}{l}\text { No specific } \\
\text { information }\end{array}$ & $\begin{array}{l}\text { Some } \\
\text { concern }\end{array}$ & $\begin{array}{l}\text { No specific } \\
\text { information }\end{array}$ & Low risk & $\begin{array}{l}\text { Predefined } \\
\text { outcomes }\end{array}$ & $\begin{array}{l}\text { Some } \\
\text { concern }\end{array}$ \\
\hline $\begin{array}{l}\text { Stepanović et al., } \\
2015 \text { [103] }\end{array}$ & $\begin{array}{l}\text { Some } \\
\text { concern }\end{array}$ & $\begin{array}{l}\text { No statement for allo- } \\
\text { cation concealment }\end{array}$ & $\begin{array}{l}\text { Some } \\
\text { concern }\end{array}$ & $\begin{array}{l}\text { Blinded only } \\
\text { in assesor }\end{array}$ & $\begin{array}{l}\text { Some } \\
\text { concern }\end{array}$ & $\begin{array}{l}\text { No specific } \\
\text { information }\end{array}$ & Low risk & Blinded & Low risk & $\begin{array}{l}\text { Predefined } \\
\text { outcomes }\end{array}$ & $\begin{array}{l}\text { Some } \\
\text { concern }\end{array}$ \\
\hline $\begin{array}{l}\text { Makharita et al., } \\
2015 \text { [102] }\end{array}$ & $\begin{array}{l}\text { Some } \\
\text { concern }\end{array}$ & $\begin{array}{l}\text { No statement for allo- } \\
\text { cation concealment }\end{array}$ & Low risk & Both blinded & Low risk & No exclusion & Low risk & Blinded & Low risk & $\begin{array}{l}\text { Predefined } \\
\text { outcomes }\end{array}$ & $\begin{array}{l}\text { Some } \\
\text { concern }\end{array}$ \\
\hline $\begin{array}{l}\text { Makharita et al., } \\
2012 \text { [101] }\end{array}$ & $\begin{array}{l}\text { Some } \\
\text { concern }\end{array}$ & $\begin{array}{l}\text { No statement for allo- } \\
\text { cation concealment }\end{array}$ & Low risk & Both blinded & Low risk & $\begin{array}{l}\text { 3/64 dropped, } \\
\text { unrelated to } \\
\text { the outcome }\end{array}$ & Low risk & Blinded & Low risk & $\begin{array}{l}\text { Predefined } \\
\text { outcomes }\end{array}$ & $\begin{array}{l}\text { Some } \\
\text { concern }\end{array}$ \\
\hline $\begin{array}{l}\text { Krcevski Skvarc } \\
\text { and Kamenik, } \\
2010 \text { [98] }\end{array}$ & High risk & $\begin{array}{l}\text { No specific informa- } \\
\text { tion }\end{array}$ & $\begin{array}{l}\text { Some } \\
\text { concern }\end{array}$ & $\begin{array}{l}\text { No specific } \\
\text { information }\end{array}$ & Low risk & No exclusion & $\begin{array}{l}\text { Some } \\
\text { concern }\end{array}$ & $\begin{array}{l}\text { No specific } \\
\text { information }\end{array}$ & Low risk & $\begin{array}{l}\text { Predefined } \\
\text { outcomes }\end{array}$ & High risk \\
\hline $\begin{array}{l}\text { Ji et al., } 2009 \\
\text { [97] }\end{array}$ & $\begin{array}{l}\text { Some } \\
\text { concern }\end{array}$ & $\begin{array}{l}\text { No statement for allo- } \\
\text { cation concealment }\end{array}$ & $\begin{array}{l}\text { Some } \\
\text { concern }\end{array}$ & $\begin{array}{l}\text { Blinded only } \\
\text { in assesor }\end{array}$ & Low risk & $\begin{array}{l}\text { 19/132 dropped, } \\
\text { propotions } \\
\text { existed }\end{array}$ & Low risk & Blinded & Low risk & $\begin{array}{l}\text { Predefined } \\
\text { outcomes }\end{array}$ & $\begin{array}{l}\text { Some } \\
\text { concern }\end{array}$ \\
\hline $\begin{array}{l}\text { van Wijck et al., } \\
2006 \text { [96] }\end{array}$ & $\begin{array}{l}\text { Some } \\
\text { concern }\end{array}$ & $\begin{array}{l}\text { No statement for allo- } \\
\text { cation concealment }\end{array}$ & $\begin{array}{l}\text { Some } \\
\text { concern }\end{array}$ & $\begin{array}{l}\text { Blinded only } \\
\text { in assesor }\end{array}$ & Low risk & $\begin{array}{l}\text { 33/598 dropped, } \\
\text { propotions } \\
\text { existed }\end{array}$ & Low risk & Blinded & Low risk & $\begin{array}{l}\text { Predefined } \\
\text { outcomes }\end{array}$ & $\begin{array}{l}\text { Some } \\
\text { concern }\end{array}$ \\
\hline $\begin{array}{l}\text { Pasqualucci et } \\
\text { al., } 2000 \text { [110] }\end{array}$ & $\begin{array}{l}\text { Some } \\
\text { concern }\end{array}$ & $\begin{array}{l}\text { No statement for allo- } \\
\text { cation concealment }\end{array}$ & $\begin{array}{l}\text { Some } \\
\text { concern }\end{array}$ & $\begin{array}{l}\text { Blinded only } \\
\text { in assesor }\end{array}$ & Low risk & $\begin{array}{l}\text { 31/600 dropped, } \\
\text { protocol viola- } \\
\text { tion }\end{array}$ & Low risk & Blinded & Low risk & $\begin{array}{l}\text { Predefined } \\
\text { outcomes }\end{array}$ & $\begin{array}{l}\text { Some } \\
\text { concern }\end{array}$ \\
\hline $\begin{array}{r}\text { Ahmed et al., } \\
1998 \text { [94] }\end{array}$ & $\begin{array}{l}\text { Some } \\
\text { concern }\end{array}$ & $\begin{array}{l}\text { No statement for allo- } \\
\text { cation concealment }\end{array}$ & $\begin{array}{l}\text { Some } \\
\text { concern }\end{array}$ & $\begin{array}{l}\text { Blinded only } \\
\text { in assesor }\end{array}$ & Low risk & No exclusion & Low risk & Blinded & Low risk & $\begin{array}{l}\text { Predefined } \\
\text { outcomes }\end{array}$ & $\begin{array}{l}\text { Some } \\
\text { concern }\end{array}$ \\
\hline $\begin{array}{l}\text { Bowsher, } 1997 \\
\text { [93] }\end{array}$ & Low risk & Sealed envelop & Low risk & Both blinded & Low risk & $\begin{array}{l}\text { 6/80 dropped, } \\
\text { unrelated to } \\
\text { the outcome }\end{array}$ & Low risk & Blinded & Low risk & $\begin{array}{l}\text { Predefined } \\
\text { outcomes }\end{array}$ & Low risk \\
\hline $\begin{array}{l}\text { Harding and } \\
\text { Porter, } 1991 \\
\text { [114] }\end{array}$ & High risk & $\begin{array}{l}\text { No statement for allo- } \\
\text { cation concealment, } \\
\text { randomization } \\
\text { sequence }\end{array}$ & $\begin{array}{l}\text { Some } \\
\text { concern }\end{array}$ & Both blinded & $\begin{array}{l}\text { Some } \\
\text { concern }\end{array}$ & $\begin{array}{l}\text { 8/46 dropped, } \\
\text { no specific } \\
\text { propotion re- } \\
\text { vealed }\end{array}$ & Low risk & Blinded & Low risk & $\begin{array}{l}\text { Predefined } \\
\text { outcomes }\end{array}$ & High risk \\
\hline $\begin{array}{c}\text { Benoldi et al., } \\
1991 \text { [92] }\end{array}$ & High risk & $\begin{array}{l}\text { No statement for allo- } \\
\text { cation concealment, } \\
\text { randomization } \\
\text { sequence }\end{array}$ & $\begin{array}{l}\text { Some } \\
\text { concern }\end{array}$ & $\begin{array}{l}\text { Different } \\
\text { procedure }\end{array}$ & Low risk & $\begin{array}{l}\text { 1/36 dropped, } \\
\text { unrelated to } \\
\text { the outcome }\end{array}$ & $\begin{array}{l}\text { Some } \\
\text { concern }\end{array}$ & $\begin{array}{l}\text { No specific } \\
\text { information }\end{array}$ & Low risk & $\begin{array}{l}\text { Predefined } \\
\text { outcomes }\end{array}$ & High risk \\
\hline $\begin{array}{c}\text { Esmann et al., } \\
1987 \text { [88] }\end{array}$ & High risk & $\begin{array}{l}\text { No statement for allo- } \\
\text { cation concealment, } \\
\text { randomization } \\
\text { sequence }\end{array}$ & $\begin{array}{l}\text { Some } \\
\text { concern }\end{array}$ & Both blinded & Low risk & No exclusion & Low risk & Blinded & Low risk & $\begin{array}{l}\text { Predefined } \\
\text { outcomes }\end{array}$ & High risk \\
\hline $\begin{array}{l}\text { Cobo et al., } 1986 \\
\text { [87] }\end{array}$ & High risk & $\begin{array}{l}\text { No statement for allo- } \\
\text { cation concealment, } \\
\text { randomization se- } \\
\text { quence, basement } \\
\text { difference existed }\end{array}$ & Low risk & Both blinded & Low risk & No exclusion & Low risk & Blinded & Low risk & $\begin{array}{l}\text { Predefined } \\
\text { outcomes }\end{array}$ & High risk \\
\hline $\begin{array}{l}\text { Balfour et al., } \\
1983 \text { [85] }\end{array}$ & $\begin{array}{l}\text { Some } \\
\text { concern }\end{array}$ & $\begin{array}{l}\text { No statement for allo- } \\
\text { cation concealment, } \\
\text { randomization } \\
\text { sequence }\end{array}$ & Low risk & Both blinded & Low risk & No exclusion & Low risk & Blinded & Low risk & $\begin{array}{l}\text { Predefined } \\
\text { outcomes }\end{array}$ & $\begin{array}{l}\text { Some } \\
\text { concern }\end{array}$ \\
\hline $\begin{array}{c}\text { Esmann et al., } \\
1982 \text { [84] }\end{array}$ & $\begin{array}{l}\text { Some } \\
\text { concern }\end{array}$ & $\begin{array}{l}\text { No statement for allo- } \\
\text { cation concealment, } \\
\text { randomization } \\
\text { sequence }\end{array}$ & Low risk & Both blinded & Low risk & 1/56 dropped & Low risk & Blinded & Low risk & $\begin{array}{l}\text { Predefined } \\
\text { outcomes }\end{array}$ & $\begin{array}{l}\text { Some } \\
\text { concern }\end{array}$ \\
\hline $\begin{array}{l}\text { Bean et al., } 1982 \\
\text { [83] }\end{array}$ & High risk & $\begin{array}{l}\text { No statement for allo- } \\
\text { cation concealment }\end{array}$ & $\begin{array}{l}\text { Some } \\
\text { concern }\end{array}$ & Both blinded & $\begin{array}{l}\text { Some } \\
\text { concern }\end{array}$ & 2/31 dropped & Low risk & Blinded & Low risk & $\begin{array}{l}\text { Predefined } \\
\text { outcomes }\end{array}$ & High risk \\
\hline $\begin{array}{l}\text { Keczkes and } \\
\text { Basheer, } 1980 \\
\text { [115] }\end{array}$ & $\begin{array}{l}\text { Some } \\
\text { concern }\end{array}$ & $\begin{array}{l}\text { No statement for allo- } \\
\text { cation concealment, } \\
\text { randomization } \\
\text { sequence }\end{array}$ & Low risk & Both blinded & Low risk & No exclusion & Low risk & Blinded & Low risk & $\begin{array}{l}\text { Predefined } \\
\text { outcomes }\end{array}$ & $\begin{array}{l}\text { Some } \\
\text { concern }\end{array}$ \\
\hline $\begin{array}{l}\text { Cui et al., } 2018 \\
\text { [107] }\end{array}$ & $\begin{array}{l}\text { Some } \\
\text { concern }\end{array}$ & $\begin{array}{l}\text { No statement for allo- } \\
\text { cation concealment }\end{array}$ & Low risk & Both blinded & Low risk & $\begin{array}{l}\text { 5/102 dropped, } \\
\text { unrelated to } \\
\text { the outcome }\end{array}$ & Low risk & Blinded & Low risk & $\begin{array}{l}\text { Predefined } \\
\text { outcomes }\end{array}$ & $\begin{array}{l}\text { Some } \\
\text { concern }\end{array}$ \\
\hline $\begin{array}{l}\text { Cui et al., } 2017 \\
\text { [105] }\end{array}$ & $\begin{array}{l}\text { Some } \\
\text { concern }\end{array}$ & $\begin{array}{l}\text { No statement for allo- } \\
\text { cation concealment, } \\
\text { randomization } \\
\text { sequence }\end{array}$ & $\begin{array}{l}\text { Some } \\
\text { concern }\end{array}$ & Both blinded & Low risk & 2/96 dropped & Low risk & Blinded & Low risk & $\begin{array}{l}\text { Predefined } \\
\text { outcomes }\end{array}$ & $\begin{array}{l}\text { Some } \\
\text { concern }\end{array}$ \\
\hline $\begin{array}{l}\text { Ni et al., } 2017 \\
\text { [106] }\end{array}$ & $\begin{array}{l}\text { Some } \\
\text { concern }\end{array}$ & $\begin{array}{l}\text { No statement for allo- } \\
\text { cation concealment }\end{array}$ & $\begin{array}{l}\text { Some } \\
\text { concern }\end{array}$ & $\begin{array}{l}\text { No specific } \\
\text { information }\end{array}$ & Low risk & $\begin{array}{l}\text { 5/100 dropped, } \\
\text { unrelated to } \\
\text { the outcome }\end{array}$ & $\begin{array}{l}\text { Some } \\
\text { concern }\end{array}$ & $\begin{array}{l}\text { No specific } \\
\text { information }\end{array}$ & Low risk & $\begin{array}{l}\text { Predefined } \\
\text { outcomes }\end{array}$ & $\begin{array}{l}\text { Some } \\
\text { concern }\end{array}$ \\
\hline
\end{tabular}


Table 2. Continued

\begin{tabular}{|c|c|c|c|c|c|c|c|c|c|c|c|}
\hline \multirow{2}{*}{$\begin{array}{l}\text { Study, year } \\
\text { Zheng et al., } \\
2019 \text { [109] }\end{array}$} & \multicolumn{2}{|c|}{ Randomization process } & \multicolumn{2}{|c|}{ Intended interventions } & \multicolumn{2}{|c|}{ Missing outcome data } & \multicolumn{2}{|c|}{$\begin{array}{l}\text { Measurement of the } \\
\text { outcome }\end{array}$} & \multicolumn{2}{|c|}{$\begin{array}{l}\text { Selection of the reported } \\
\text { result }\end{array}$} & \multirow{2}{*}{$\begin{array}{l}\begin{array}{c}\text { Overall } \\
\text { result }\end{array} \\
\text { Some } \\
\text { concern }\end{array}$} \\
\hline & $\begin{array}{l}\text { Some } \\
\text { concern }\end{array}$ & $\begin{array}{l}\text { No statement for allo- } \\
\text { cation concealment }\end{array}$ & Low risk & Both blinded & Low risk & 13/153 dropped & Low risk & Blinded & Low risk & $\begin{array}{l}\text { Predefined } \\
\text { outcomes }\end{array}$ & \\
\hline $\begin{array}{l}\text { Hwang et al., } \\
1999 \text { [79] }\end{array}$ & High risk & $\begin{array}{l}\text { No statement for allo- } \\
\text { cation concealment, } \\
\text { randomization } \\
\text { sequence }\end{array}$ & $\begin{array}{l}\text { Some } \\
\text { concern }\end{array}$ & $\begin{array}{l}\text { No specific } \\
\text { information }\end{array}$ & Low risk & No exclusion & $\begin{array}{l}\text { Some } \\
\text { concern }\end{array}$ & $\begin{array}{l}\text { No specific } \\
\text { information }\end{array}$ & Low risk & $\begin{array}{l}\text { Predefined } \\
\text { outcomes }\end{array}$ & High risk \\
\hline $\begin{array}{l}\text { Wan et al., } 2019 \\
\text { [120] }\end{array}$ & $\begin{array}{l}\text { Some } \\
\text { concern }\end{array}$ & $\begin{array}{l}\text { No statement for allo- } \\
\text { cation concealment }\end{array}$ & $\begin{array}{l}\text { Some } \\
\text { concern }\end{array}$ & Both blinded & Low risk & $\begin{array}{l}\text { 2/96 dropped, } \\
\text { unrelated to } \\
\text { the outcome }\end{array}$ & Low risk & Blinded & Low risk & $\begin{array}{l}\text { Predefined } \\
\text { outcomes }\end{array}$ & $\begin{array}{l}\text { Some } \\
\text { concern }\end{array}$ \\
\hline $\begin{array}{l}\text { Hügler et al., } \\
2002 \text { [116] }\end{array}$ & High risk & $\begin{array}{l}\text { No statement for allo- } \\
\text { cation concealment, } \\
\text { randomization } \\
\text { sequence }\end{array}$ & $\begin{array}{l}\text { Some } \\
\text { concern }\end{array}$ & Both blinded & Low risk & No exclusion & Low risk & Blinded & Low risk & $\begin{array}{l}\text { Predefined } \\
\text { outcomes }\end{array}$ & High risk \\
\hline $\begin{array}{l}\text { Cui et al., } 2016 \\
\text { [117] }\end{array}$ & $\begin{array}{l}\text { Some } \\
\text { concern }\end{array}$ & $\begin{array}{l}\text { No statement for allo- } \\
\text { cation concealment, } \\
\text { randomization } \\
\text { sequence }\end{array}$ & $\begin{array}{l}\text { Some } \\
\text { concern }\end{array}$ & $\begin{array}{l}\text { No specific } \\
\text { information }\end{array}$ & Low risk & No exclusion & Low risk & Blinded & Low risk & $\begin{array}{l}\text { Predefined } \\
\text { outcomes }\end{array}$ & $\begin{array}{l}\text { Some } \\
\text { concern }\end{array}$ \\
\hline $\begin{array}{l}\text { Payne et al., } \\
1989 \text { [91] }\end{array}$ & $\begin{array}{l}\text { Some } \\
\text { concern }\end{array}$ & $\begin{array}{l}\text { No statement for allo- } \\
\text { cation concealment }\end{array}$ & Low risk & Both blinded & Low risk & 3/41 dropped & Low risk & Blinded & Low risk & $\begin{array}{l}\text { Predefined } \\
\text { outcomes }\end{array}$ & $\begin{array}{l}\text { Some } \\
\text { concern }\end{array}$ \\
\hline $\begin{array}{l}\text { Wood et al., } 1994 \\
\text { [111] }\end{array}$ & $\begin{array}{l}\text { Some } \\
\text { concern }\end{array}$ & $\begin{array}{l}\text { No statement for allo- } \\
\text { cation concealment }\end{array}$ & Low risk & Both blinded & Low risk & $\begin{array}{l}\text { 51/400 dropped, } \\
\text { unrelated to } \\
\text { the outcome }\end{array}$ & Low risk & Blinded & Low risk & $\begin{array}{l}\text { Predefined } \\
\text { outcomes }\end{array}$ & $\begin{array}{l}\text { Some } \\
\text { concern }\end{array}$ \\
\hline $\begin{array}{l}\text { McGill et al., } \\
1983 \text { [86] }\end{array}$ & $\begin{array}{l}\text { Some } \\
\text { concern }\end{array}$ & $\begin{array}{l}\text { No statement for allo- } \\
\text { cation concealment }\end{array}$ & Low risk & Both blinded & Low risk & $\begin{array}{l}3 / 40 \text { dropped, } \\
\text { unrelated to } \\
\text { the outcome }\end{array}$ & Low risk & Blinded & Low risk & $\begin{array}{l}\text { Predefined } \\
\text { outcomes }\end{array}$ & $\begin{array}{l}\text { Some } \\
\text { concern }\end{array}$ \\
\hline $\begin{array}{c}\text { Wassilew et al., } \\
1987 \text { [89] }\end{array}$ & $\begin{array}{l}\text { Some } \\
\text { concern }\end{array}$ & $\begin{array}{l}\text { No statement for allo- } \\
\text { cation concealment, } \\
\text { randomization } \\
\text { sequence }\end{array}$ & Low risk & Both blinded & Low risk & $2 / 60$ dropped & Low risk & Blinded & Low risk & $\begin{array}{l}\text { Predefined } \\
\text { outcomes }\end{array}$ & $\begin{array}{l}\text { Some } \\
\text { concern }\end{array}$ \\
\hline $\begin{array}{c}\text { Mandal et al., } \\
1988 \text { [90] }\end{array}$ & High risk & $\begin{array}{l}\text { No statement for allo- } \\
\text { cation concealment, } \\
\text { randomization se- } \\
\text { quence, basement } \\
\text { difference existed }\end{array}$ & Low risk & Both blinded & Low risk & $\begin{array}{c}8 / 64 \text { dropped, } \\
\text { unrelated to } \\
\text { the outcome }\end{array}$ & Low risk & Blinded & Low risk & $\begin{array}{l}\text { Predefined } \\
\text { outcomes }\end{array}$ & High risk \\
\hline $\begin{array}{l}\text { Lee et al., } 1999 \\
\text { [95] }\end{array}$ & High risk & $\begin{array}{l}\text { No statement for allo- } \\
\text { cation concealment, } \\
\text { randomization se- } \\
\text { quence, basement } \\
\text { difference existed }\end{array}$ & $\begin{array}{l}\text { Some } \\
\text { concern }\end{array}$ & $\begin{array}{l}\text { No specific } \\
\text { information }\end{array}$ & Low risk & No exclusion & Low risk & Blinded & Low risk & $\begin{array}{l}\text { Predefined } \\
\text { outcomes }\end{array}$ & High risk \\
\hline $\begin{array}{l}\text { Harding et al., } \\
1986 \text { [119] }\end{array}$ & $\begin{array}{l}\text { Some } \\
\text { concern }\end{array}$ & $\begin{array}{l}\text { No statement for allo- } \\
\text { cation concealment }\end{array}$ & $\begin{array}{l}\text { Some } \\
\text { concern }\end{array}$ & $\begin{array}{l}\text { No specific } \\
\text { information }\end{array}$ & $\begin{array}{l}\text { Some } \\
\text { concern }\end{array}$ & $\begin{array}{l}\text { No specific } \\
\text { information }\end{array}$ & $\begin{array}{l}\text { Some } \\
\text { concern }\end{array}$ & $\begin{array}{l}\text { No specific } \\
\text { information }\end{array}$ & Low risk & $\begin{array}{l}\text { Predefined } \\
\text { outcomes }\end{array}$ & $\begin{array}{l}\text { Some } \\
\text { concern }\end{array}$ \\
\hline $\begin{array}{l}\text { Kanodia and } \\
\text { Singhal, } 2011 \\
\text { [99] }\end{array}$ & $\begin{array}{l}\text { Some } \\
\text { concern }\end{array}$ & $\begin{array}{l}\text { No statement for allo- } \\
\text { cation concealment, } \\
\text { randomization } \\
\text { sequence }\end{array}$ & Low risk & Both blinded & $\begin{array}{l}\text { Some } \\
\text { concern }\end{array}$ & $\begin{array}{l}\text { No specific } \\
\text { information }\end{array}$ & Low risk & Blinded & Low risk & $\begin{array}{l}\text { Predefined } \\
\text { outcomes }\end{array}$ & $\begin{array}{l}\text { Some } \\
\text { concern }\end{array}$ \\
\hline $\begin{array}{l}\text { Kanodia et al., } \\
2012 \text { [100] }\end{array}$ & $\begin{array}{l}\text { Some } \\
\text { concern }\end{array}$ & $\begin{array}{l}\text { No statement for allo- } \\
\text { cation concealment, } \\
\text { randomization } \\
\text { sequence }\end{array}$ & Low risk & Both blinded & $\begin{array}{l}\text { Some } \\
\text { concern }\end{array}$ & $\begin{array}{l}\text { No specific } \\
\text { information }\end{array}$ & Low risk & Blinded & Low risk & $\begin{array}{l}\text { Predefined } \\
\text { outcomes }\end{array}$ & $\begin{array}{l}\text { Some } \\
\text { concern }\end{array}$ \\
\hline $\begin{array}{l}\text { Manabe et al., } \\
2004 \text { [118] }\end{array}$ & High risk & $\begin{array}{l}\text { No statement for allo- } \\
\text { cation concealment, } \\
\text { randomization se- } \\
\text { quence, basement } \\
\text { difference existed }\end{array}$ & Low risk & Both blinded & Low risk & $\begin{array}{l}\text { 3/59 dropped, } \\
\text { unrelated to } \\
\text { the outcome }\end{array}$ & Low risk & Blinded & Low risk & $\begin{array}{l}\text { Predefined } \\
\text { outcomes }\end{array}$ & High risk \\
\hline $\begin{array}{l}\text { Clemmensen } \\
\text { and Andersen, } \\
1984 \text { [112] }\end{array}$ & High risk & $\begin{array}{l}\text { No statement for allo- } \\
\text { cation concealment, } \\
\text { randomization se- } \\
\text { quence, basement } \\
\text { difference existed }\end{array}$ & Low risk & Both blinded & Low risk & $\begin{array}{l}\text { 5/60 dropped, } \\
\text { unrelated to } \\
\text { outcome }\end{array}$ & Low risk & Blinded & Low risk & $\begin{array}{l}\text { Predefined } \\
\text { outcomes }\end{array}$ & High risk \\
\hline $\begin{array}{c}\text { Eaglstein et al., } \\
1970 \text { [113] }\end{array}$ & High risk & $\begin{array}{l}\text { No statement for allo- } \\
\text { cation concealment, } \\
\text { randomization } \\
\text { sequence }\end{array}$ & Low risk & Both blinded & $\begin{array}{l}\text { Some } \\
\text { concern }\end{array}$ & 1/35 dropped & Low risk & Blinded & Low risk & $\begin{array}{l}\text { Predefined } \\
\text { outcomes }\end{array}$ & High risk \\
\hline
\end{tabular}

suggested a less likely publication bias (Fig. 6A). The network diagnostics, using trace and density plots, showed that model convergence was valid in both fixed and ran- dom-effects models (Supplementary Figs. 1D, 1E). However, the Gelman-Rubin-Brooks methods with PSRF and DIC showed that the random-effects model is a slightly 
A

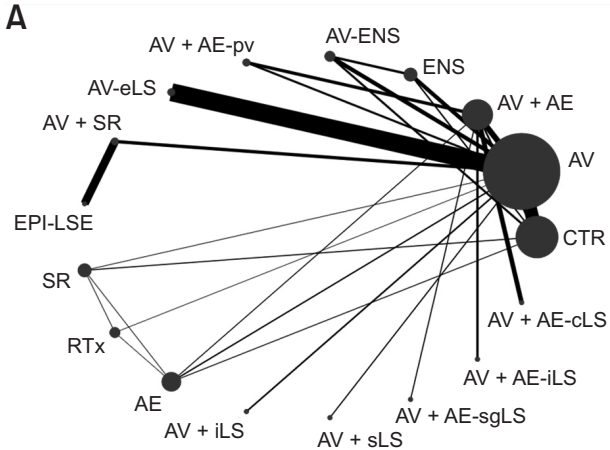

C

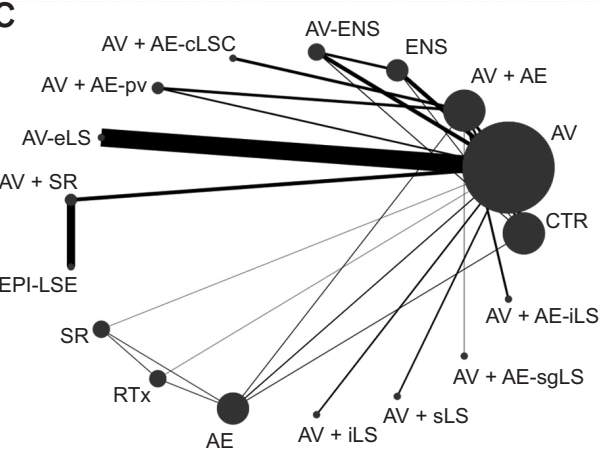

E

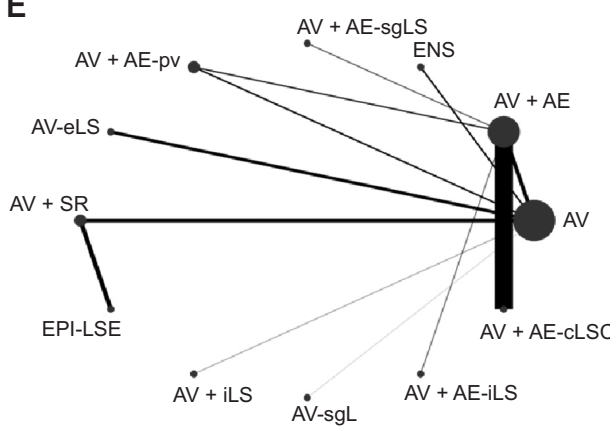

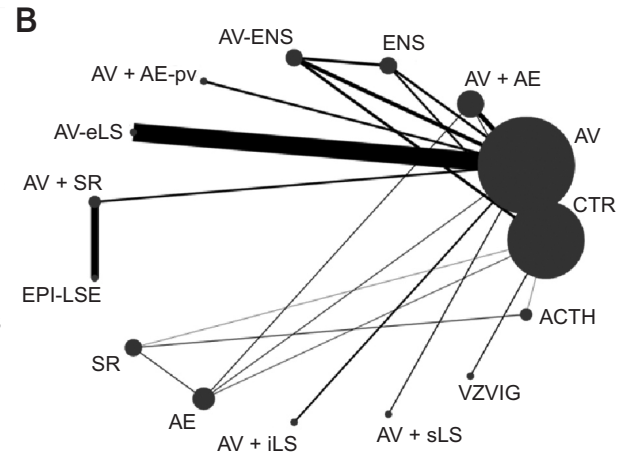

D

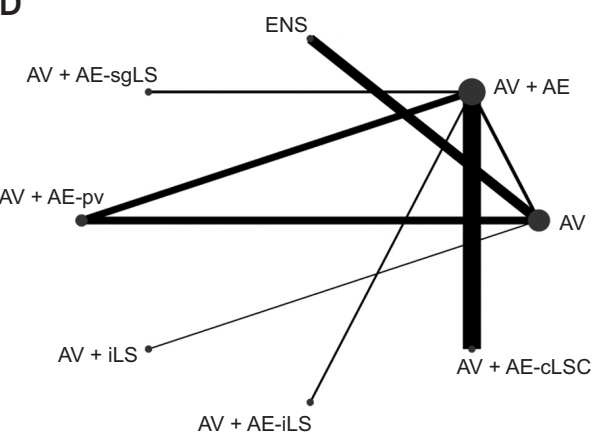

F

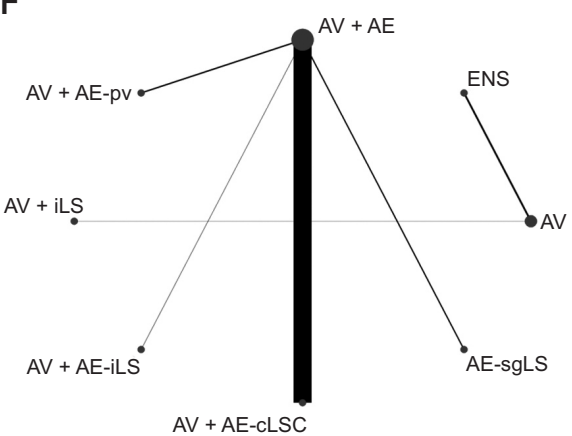

Fig. 2. Network plot of included studies. The nodes show each strategy and the edges show the available direct comparisons among the strategies. The nodes and edges are weighed based on the number of included patients and inverse of standard error of effect. (A) The incidence of postherpetic neuralgia at 3 months after acute herpes zoster, (B) the incidence of postherpetic neuralgia at 1 month after acute herpes zoster, (C) the incidence of postherpetic neuralgia at 6 months after acute herpes zoster, (D) pain score measured at 3 months after acute herpes zoster, (E) pain score measured at 1 month after acute herpes zoster, and (F) pain score measured at 6 months after acute herpes zoster. CTR: control, RTx: radiotherapy, VZVIG: varicella zoster vaccine immunoglobulin, ACTH: adrenocorticotrophic hormone. better fit for the data (Supplementary Figs. 1F, 1G, Supplementary Table 1). Heterogeneity was also decreased in random-effects model compared with fixed-effects model (Supplementary Table 1). Thus, we analyzed the data using a random-effects model.

The SUCRA values from the Bayesian model were similar to those from the frequentist model (Fig. 7A). When performing a meta-regression analysis using the risk of bias (low risk vs. some concern or high risk), age and sex did not improve the model fit or substantially decrease the heterogeneity, and were not considered statistically significant (Supplementary Table 3).

\section{Incidence of PHN at 1 month after acute HZ}

A total of 21 studies (2,612 patients) measured the incidence of PHN at 1 month after acute HZ. Of these, two studies were separated from the loops [117,118]. We performed an NMA excluding those studies. Thus, a total of 19 studies (2,492 patients) were analyzed. The network plot of all eligible comparisons for this endpoint is shown in Fig. 2B. Although all 15 management modalities (nodes) were connected to the network, two comparisons (AV and CTR) were more directly comparable than the other 13 nodes. No network inconsistencies were observed $\left[\chi^{2}(4)=7.97, P=\right.$ 0.093].

Ten closed loops were identified in the network after comparing the incidence of PHN at 1 month after acute HZ. Four loops (CTR/AV + AE/AE [93], CTR/ENS/AV-ENS [103], CTR/SR/ACTH [112], and AV/ENS/AV-ENS [103]) were formed only by multi-arm trials. Of the six closed loops, inconsistencies were observed in 1/10/11 (CTR/SR/AE), and 1/2/3 (CTR/AV/AV + AE) (Fig. 3B). EPI-LSE, AV + iLS, AV + AE-pV, AV + sLS, and AV + SR showed a lower incidence of PHN at 1 month than CTR, only in terms of 95\% CI not in PrI (Fig. 4B, Supplementary Fig. 2A, Supplementary Table 1B). The rankograms showed that EPI-LSE followed by AV + AE-pV had the lowest incidence of PHN at 1 month after 


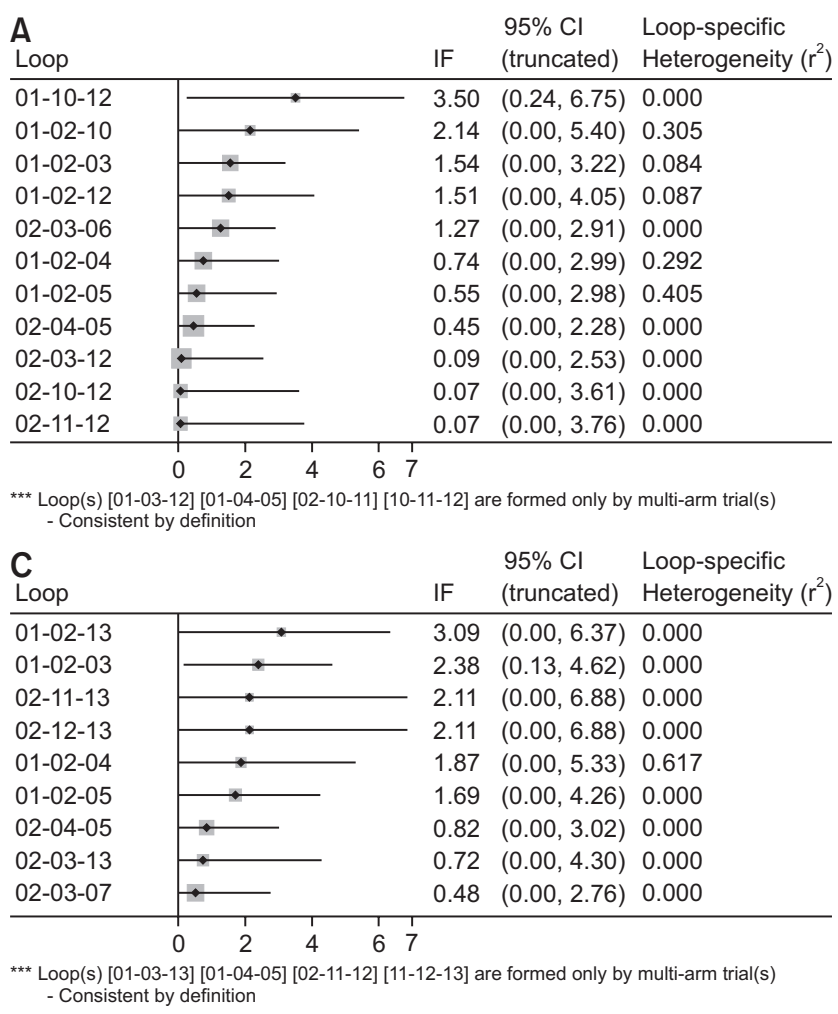

\begin{tabular}{|c|c|c|c|c|}
\hline \multicolumn{2}{|l|}{$\begin{array}{l}\text { B } \\
\text { Loop }\end{array}$} & \multirow{2}{*}{ IF } & \multirow{2}{*}{$\begin{array}{l}95 \% \mathrm{Cl} \\
\text { (truncated) }\end{array}$} & \multirow{2}{*}{$\begin{array}{l}\text { Loop-specific } \\
\text { Heterogeneity }\left(r^{2}\right) \\
0.000\end{array}$} \\
\hline $01-10-11$ & $\longrightarrow$ & & & \\
\hline $01-02-03$ & $\rightarrow$ & 1.94 & $(0.30,3.58)$ & 0.000 \\
\hline $01-02-11$ & $\rightarrow$ & 1.75 & $(0.00,4.23)$ & 0.000 \\
\hline 02-03-11 & $1-$ & 0.26 & $(0.00,2.60)$ & 0.000 \\
\hline 01-02-05 & $\frac{1}{-1}$ & 0.02 & $(0.00,1.92)$ & 0.240 \\
\hline 01-02-04 & $\div$ & 0.02 & $(0.00,2.07)$ & 0.240 \\
\hline \multicolumn{5}{|c|}{\begin{tabular}{l|llll} 
& 1 & 1 & 1 \\
& 0 & 3579
\end{tabular}} \\
\hline \multicolumn{5}{|c|}{$\begin{array}{l}\text { *** Loop(s) [01-03-11] [01-04-05] [01-10-15] [02-04-05] are formed only by multi-arm trial(s) } \\
\text { - Consistent by definition }\end{array}$} \\
\hline $\begin{array}{l}\text { D } \\
\text { Loop }\end{array}$ & & IF & $\begin{array}{l}95 \% \mathrm{Cl} \\
\text { (truncated) }\end{array}$ & $\begin{array}{l}\text { Loop-specific } \\
\text { Heterogeneity }\left(r^{2}\right)\end{array}$ \\
\hline \multirow[t]{2}{*}{$1-2-5$} & $\longrightarrow$ & 0.51 & $(0.00,1.34)$ & 0.000 \\
\hline & $\begin{array}{lll}1 & 1 & 1\end{array}$ & & & \\
\hline $\begin{array}{l}\text { E } \\
\text { Loop }\end{array}$ & & IF & $\begin{array}{l}95 \% \mathrm{Cl} \\
\text { (truncated) }\end{array}$ & $\begin{array}{l}\text { Loop-specific } \\
\text { Heterogeneity }\left(r^{2}\right)\end{array}$ \\
\hline $01-02-05$ & $\longrightarrow$ & 1.04 & $(0.24,1.84)$ & 0.000 \\
\hline
\end{tabular}

Fig. 3. Inconsistency plot between the direct and indirect effect estimates for the same comparison. Inconsistency factor (IF) as the absolute difference with $95 \%$ confidence interval $(\mathrm{Cl})$ between the direct and indirect estimates for each paired comparison is presented. IF values close to 0 indicate that the two sources are in agreement. (A) The incidence of postherpetic neuralgia at 3 months after acute herpes zoster, (B) the incidence of postherpetic neuralgia at 1 month after acute herpes zoster, $(C)$ the incidence of postherpetic neuralgia at 6 months after acute herpes zoster, (D) pain score measured at 3 months after acute herpes zoster, and (E) pain score measured at 1 month after acute herpes zoster.

acute HZ (Supplementary Fig. 2B). A cumulative ranking plot was drawn, and the SUCRA probabilities of the different interventions for PHN at 1 month after acute HZ were calculated (Supplementary Fig. 2C).

According to the SUCRA values, the incidence of PHN at 1 month after acute $\mathrm{HZ}$ was lower in the order of the EPI-LSE (98.7\%), followed by AV + AE-pV (81.4\%), AV + iLS (80.9\%), and AV + sLS (77.0\%) (Fig. 5B). The comparisonadjusted funnel plots showed that the funnel plots were symmetrical around the zero line, suggesting a lesser probability for publication bias (Fig. 6B).

The network diagnostics using trace and density plots showed that model convergence was valid in both fixedeffects and random-effects models (Supplementary Figs. 2D, 2E). However, the Gelman-Rubin-Brooks method with the PSRF and DIC showed that the random-effects model is a slightly better fit for the data (Supplementary Figs. 2F, 2G, Supplementary Table 2). Thus, we analyzed the data using a random-effects model. The SUCRA values from the Bayesian model were similar to those from the frequentist model, demonstrating the robustness of our analysis (Fig. 7B). When performing a meta-regression analysis using the risk of bias (low risk vs. some concern or high risk), age and sex did not improve the model fit or substantially de- crease the heterogeneity, and were not considered statistically significant (Supplementary Table 3).

\section{Incidence of PHN at 6 months after acute HZ}

A total of 17 studies (2,502 patients) measured the incidence of PHN at 6 months after acute HZ. Although all 17 management modalities (nodes) were connected to the network, three comparisons (AV, AV + AE and CTR) were more directly comparable than the other 14 nodes (Fig. 2C). There was no evidence of network inconsistency $\left[\chi^{2}\right.$ (6) $=8.72, P=0.190]$. Thirteen closed loops were identified in the network after comparing the incidence of PHN at 6 months after acute HZ, but four loops (CTR/AV + AE/AE [93], CTR/ENS/AV-ENS [103], AV/SR/RTx [92], and SR/RTx/ $\mathrm{AE}$ [92]) were formed only by multi-arm trials. Of the six closed loops, inconsistencies were observed in the $1 / 2 / 3$ loop (CTR/AV/AV + AE) (Fig. 3C). AV + AE-pv showed a lower incidence of PHN at 6 months after acute HZ than CTR, only in terms of 95\% CI (Fig. 4C, Supplementary Fig. 3A, Supplementary Table 1C). The AV + AE-cLSC showed marginal significance.

The rankograms and cumulative ranking plot showed that AV + AE-sgLS, AV + AE-cLSC, AV + AE-pv, and AV + 
A

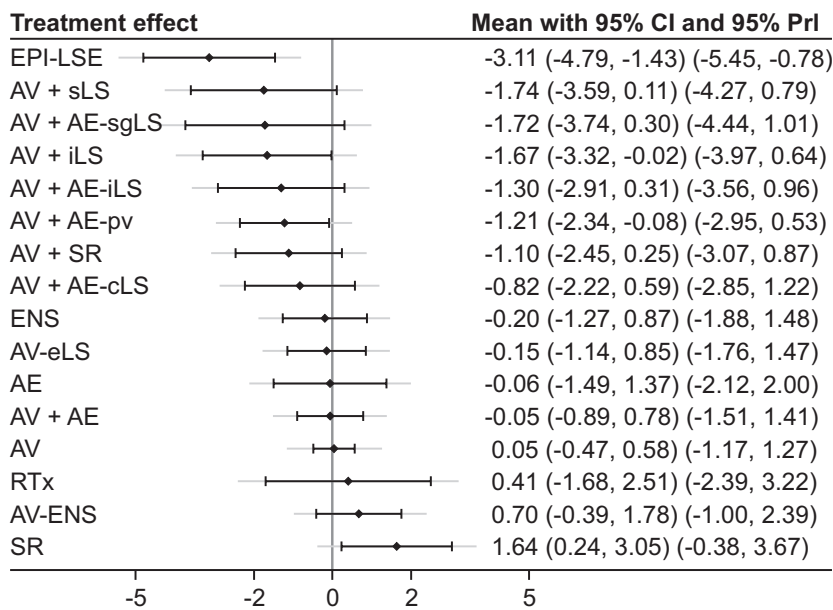

C Reference treatment: CTR

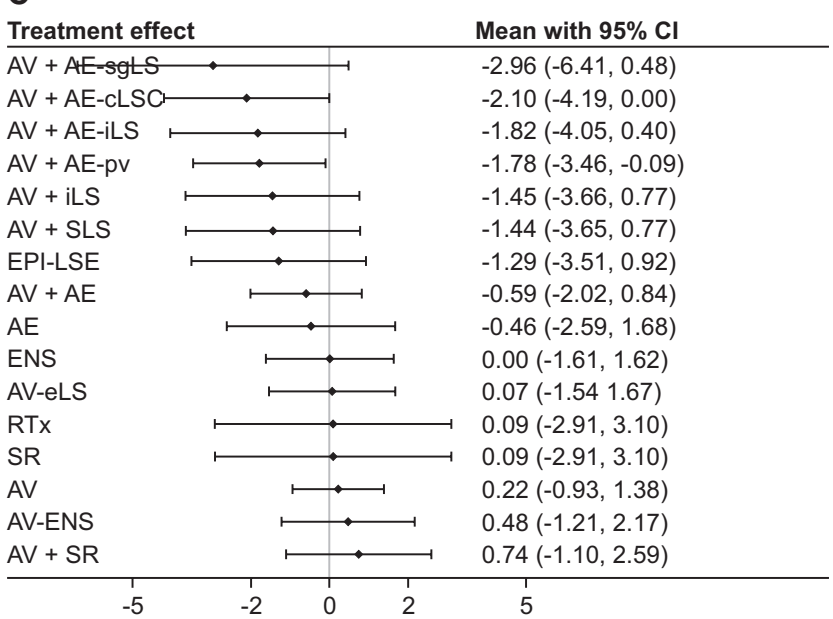

AE-iLS had the lowest incidence of PHN at 6 months after acute HZ (Supplementary Figs. 3B, 3C).

According to the SUCRA value, the incidence of PHN at 6 months after acute $\mathrm{HZ}$ was lower in the order of the $\mathrm{AV}+\mathrm{AE}-\operatorname{sgLS}(87.8 \%)$, followed by AV + AE-cLSC (81.7\%), AV + AE-pv (77.9\%), and AV + AE-iLS (76.0\%) (Fig. 5C). The comparison-adjusted funnel plots showed that the funnel plots were symmetrical around the zero line, suggesting a lesser probability for publication bias (Fig. 6C). The network diagnostics, using trace and density plots, showed that model convergence was valid in both fixed-effects and random-effects models (Supplementary Figs. 3D, 3E). However, the Gelman-Rubin-Brooks methods with PSRF and DIC showed that the random-effects model is a slightly better fit for the data (Supplementary Figs. 3F, 3G, Supplementary Table 2). Thus, we analyzed the data using a random-effects model.

The SUCRA values from the Bayesian model were similar to those from the frequentist model, demonstrating the robustness of our analysis (Fig. 7C). When performing a meta-regression analysis using the risk of bias (low risk vs.

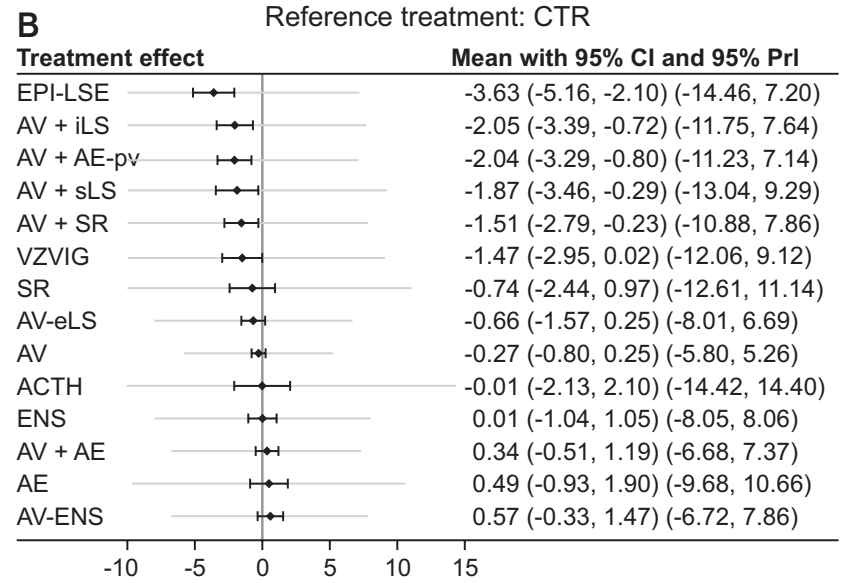

Fig. 4. Predictive interval plots (Prls) compared with control. A diamond shape represents the mean summary effects. The black line represents the 95\% confidence interval $(\mathrm{Cl})$, and the red line represents the Prl. Prls provide an interval that is expected to encompass the estimate of a future study. (A) The incidence of postherpetic neuralgia at 3 months after acute herpes zoster, (B) the incidence of postherpetic neuralgia at 1 month after acute herpes zoster, and $(C)$ the incidence of postherpetic neuralgia at 6 months after acute herpes zoster. CTR: control, RTx: radiotherapy, VZVIG: varicella zoster vaccine immunoglobulin, $\mathrm{ACTH}$ : adrenocorticotrophic hormone.

some concern or high risk), age and sex did not improve the model fit or substantially decrease the heterogeneity, and were not considered statistically significant (Supplementary Table 3).

\section{Pain score measured at 3 months after acute $\mathrm{HZ}$}

A total of 14 studies (2,047 patients) measured the pain score at 3 months after acute HZ. Of these, three studies $[104,114,119]$ did not report any information on the degree of scattering. Thus, we attempted to contact the study authors but could not obtain information on the degree of scattering. As two studies were separated from the loops $[110,120]$, we performed an NMA, excluding those studies. Thus, only nine studies (1,225 patients) were analyzed. The network plot of all eligible comparisons for this endpoint is shown in (Fig. 2D). Eight management modalities (nodes) were connected to the network. One closed loop (the $1 / 2 / 5$ loop) (AV/AV + AE/AV + AE-pv) was identified in the network after comparing the pain score at 3 months after acute HZ, which shows no evidence of inconsistency 

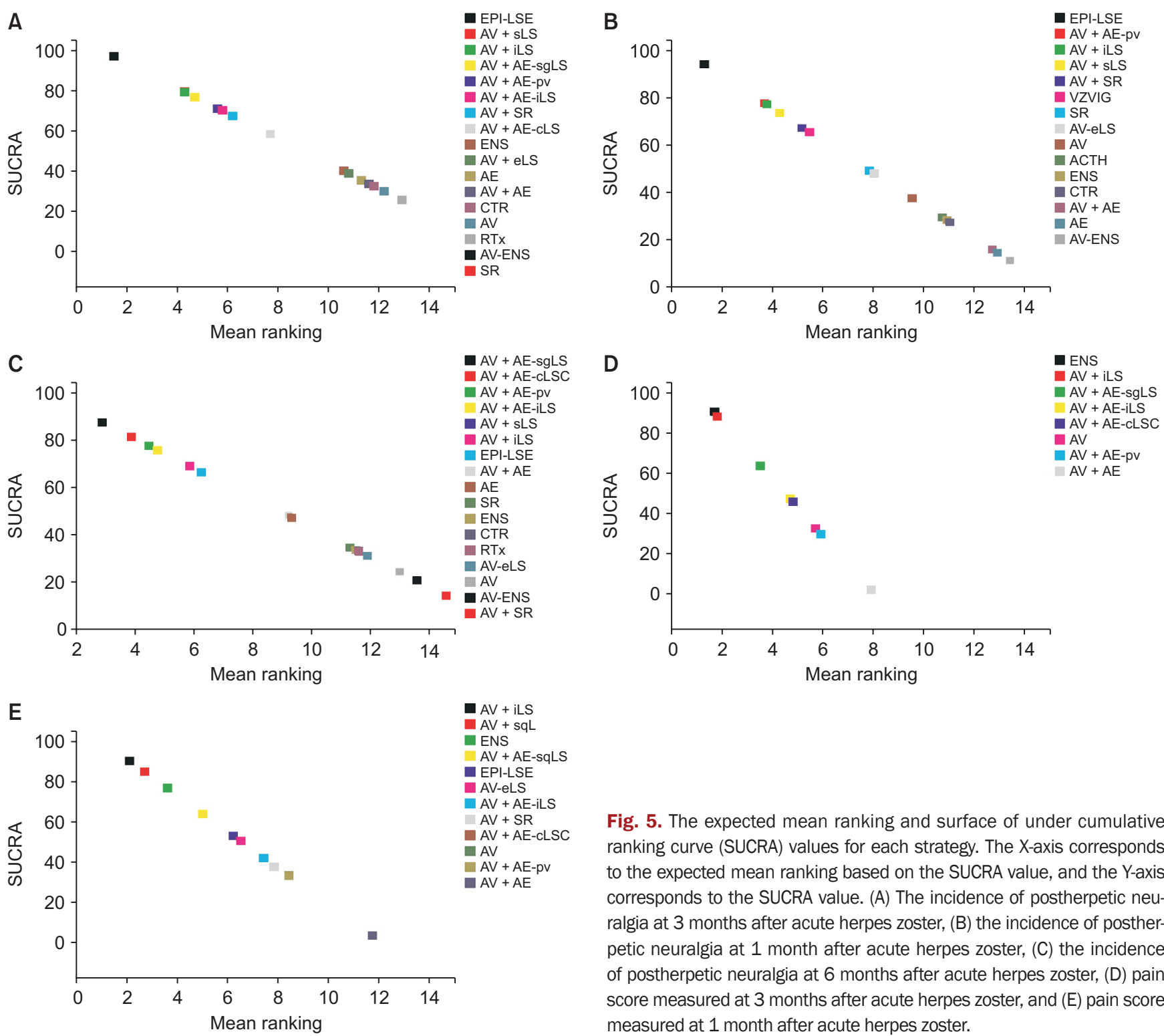

(Fig. 3D).

A PrI plot and league table comparing all groups were drawn (Supplementary Fig. 4A, Supplementary Table 1D). The rankograms and cumulative ranking plot showed that $\mathrm{AV}+\mathrm{iLS}$ and ENS had the lowest pain score at 3 months (Supplementary Figs. 4B, 4C). According to the SUCRA values, the pain score at 3 months was lower in the order of the ENS (93.0\%) followed by AV + iLS (91.0\%), followed by $\mathrm{AV}+\mathrm{AE}-\mathrm{sgLS}$ (65.9\%) (Fig. 4E). The comparison-adjusted funnel plots showed that the funnel plots were symmetrical around the zero line, which suggested a lesser probability for publication bias (Fig. 6D). The network diagnostics using trace and density plots showed that model convergence was valid in both fixed-effects and randomeffects models (Supplementary Figs. 4D, 4E). However, the Gelman-Rubin-Brooks methods with PSRF and DIC

showed that the random-effects model is a slightly better fit for the data (Supplementary Figs. 4F, 4G, Supplementary Table 2). Thus, we analyzed data using random-effects model.

The SUCRA values from the Bayesian model were similar to those from the frequentist model, demonstrating the robustness of our analysis (Fig. 7D). When performing a meta-regression analysis using the risk of bias (low risk vs. some concern or high risk), age and sex did not improve the model fit or substantially decrease the heterogeneity, and were not considered statistically significant (Supplementary Table 3).

\section{Pain score measured at 1 month after acute HZ}

A total of 21 studies $(2,758$ patients $)$ measured the pain 


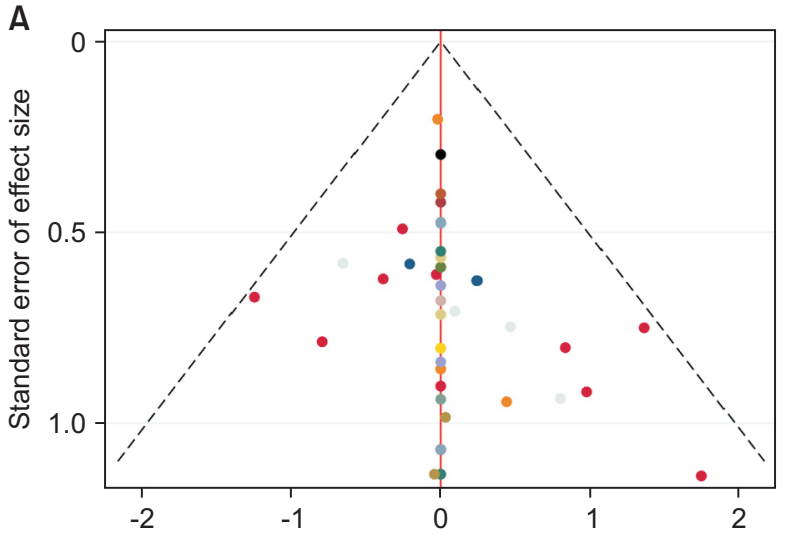

Effect size centred at comparison-specific pooled effect $\left(y_{i x y}-\mu x y\right)$

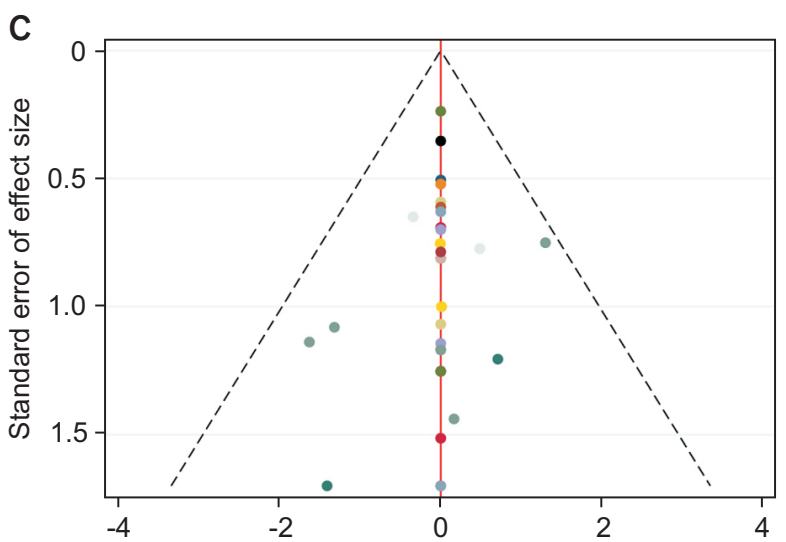

Effect size centred at comparison-specific pooled effect $\left(y_{i x y}-\mu x y\right)$

E

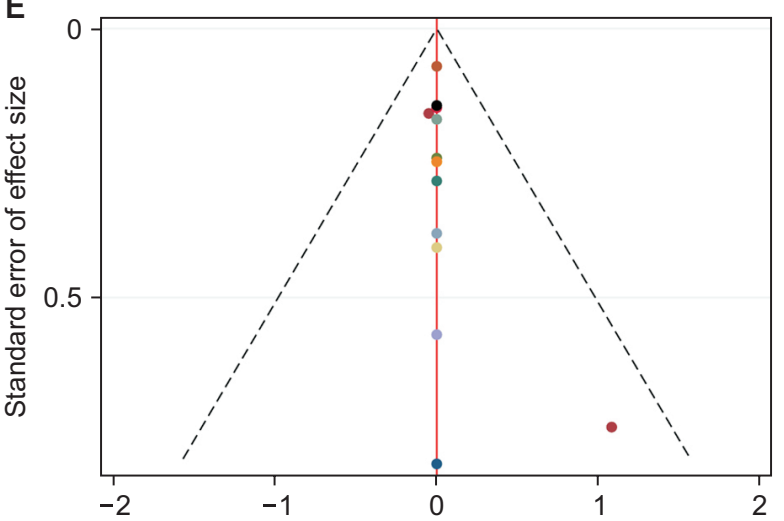

Effect size centred at comparison-specific pooled effect $\left(y_{i x y}-\mu x y\right)$

score at 1 month after acute HZ. Of these, three studies $[104,114,119]$ did not report any information on the degree of scattering. Thus, we attempted to contact the study authors, but could not obtain information on the degree of scattering. As three studies were separated from the loops $[116,117,120]$, we performed NMA excluding those studies. Thus, only 15 studies (2,385 patients) were analyzed. The network plot of all eligible comparisons for this endpoint is shown in (Fig. 2E). Although all 12 management modalities (nodes) were connected to the network, two comparisons ( $\mathrm{AV}$ and $\mathrm{AV}+\mathrm{RE}$ ) were more directly comparable than the other 10 nodes.

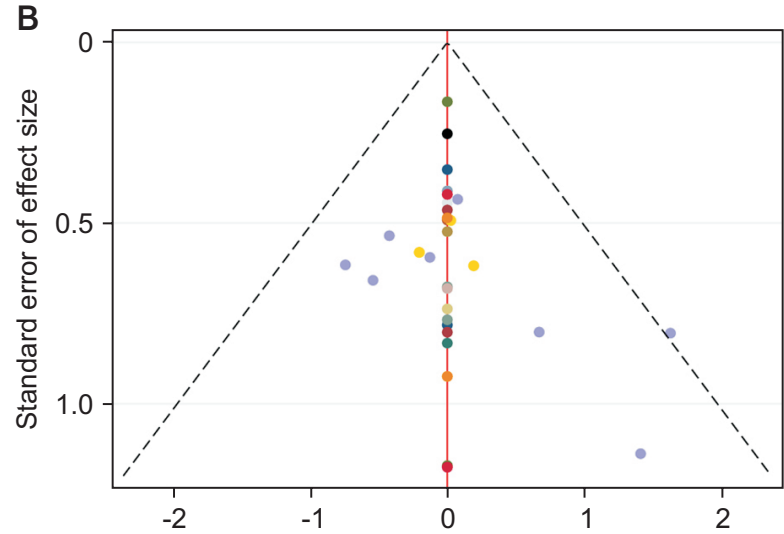

Effect size centred at comparison-specific pooled effect $\left(y_{i x y}-\mu x y\right)$

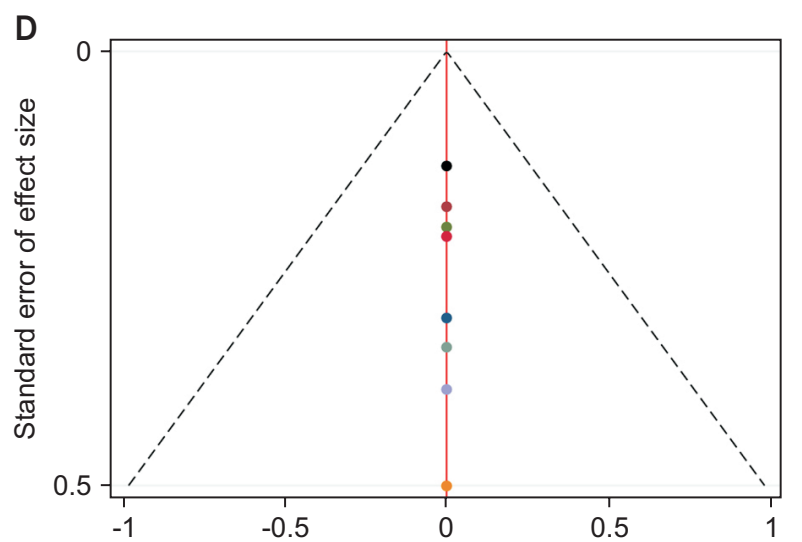

Effect size centred at comparison-specific pooled effect $\left(y_{i x y}-\mu x y\right)$

Fig. 6. Comparison-adjusted funnel plot. (A) The incidence of postherpetic neuralgia at 3 months after acute herpes zoster, $(B)$ the incidence of postherpetic neuralgia at 1 month after acute herpes zoster, $(C)$ the incidence of postherpetic neuralgia at 6 months after acute herpes zoster, (D) pain score measured at 3 months after acute herpes zoster, and $(E)$ pain score measured at 1 month after acute herpes zoster.

There was no evidence of network inconsistency $\left[\chi^{2}(1)\right.$ $=0.14, P=0.713]$. One closed loop (the 1/2/5 loop) (AV/AV + $\mathrm{AE} / \mathrm{AV}+\mathrm{AE}-\mathrm{pv}$ ) was identified in the network after comparing the pain scores at 1 month after acute HZ, which showed inconsistencies in this loop (Fig. 3E).

A PrI plot and league table, comparing all groups, were drawn (Supplementary Figs. 5A, Supplemeantary Table 1E).

The rankograms and cumulative ranking plot showed that $\mathrm{AV}+\mathrm{iLS}$ and $\mathrm{AV}+\mathrm{AE}$-sqLS had the lowest pain score at 1 month (Supplementary Figs. 5B, 5C). According to the SUCRA values, the pain score at 1 month was lower in the 

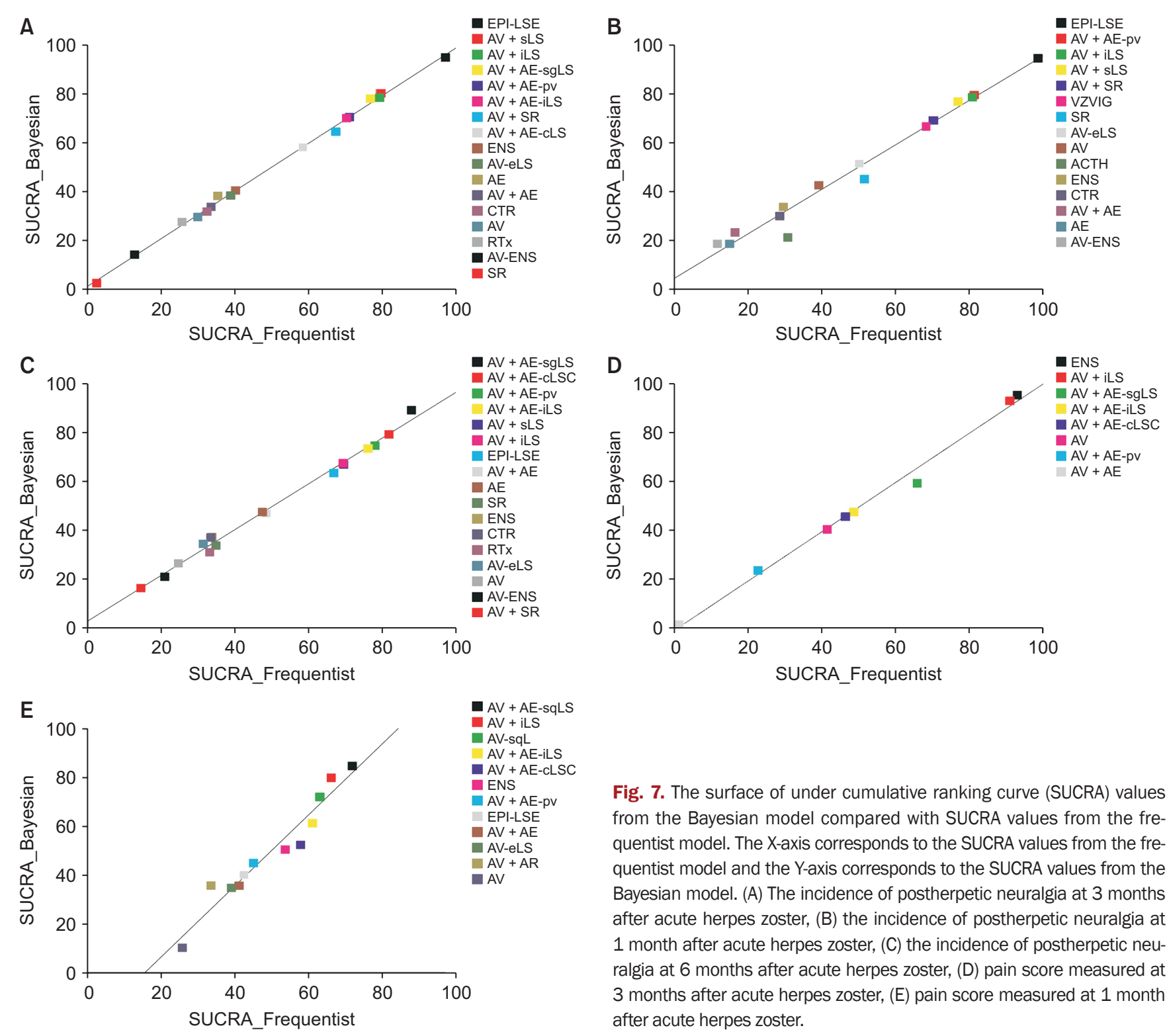

order of the AV + AE-sgLS (71.8\%), followed by AV + iLSL (66.1\%), AV-sgL (63.0\%), and AV + AE-iLS (61.0\%) (Fig. 5E). The comparison-adjusted funnel plots showed that the funnel plots were symmetrical around the zero line, suggesting a lesser probability for publication bias (Fig. 6E).

The network diagnostics, using trace and density plots, showed that model convergence was valid in both fixedeffects and random-effects model (Supplementary Figs. 5D, 5E). However, the Gelman-Rubin-Brooks methods with PSRF and DIC showed that the random-effects model is a slightly better fit for the data (Supplementary Figs. 5F, 5G, Supplementary Table 2). Thus, we analyzed data using the random-effects model.

The SUCRA values from the Bayesian model were similar to those from the frequentist model, demonstrating the robustness of our analysis (Fig. 7E). 
Table 3. The GRADE evidence quality for each outcome

\begin{tabular}{|c|c|c|c|c|c|c|c|c|}
\hline & \multirow{2}{*}{$\begin{array}{l}\text { Number of } \\
\text { studies }\end{array}$} & \multirow{2}{*}{$\begin{array}{l}\text { Number of } \\
\text { patients }\end{array}$} & \multicolumn{5}{|c|}{ Quality assessment } & \multirow[b]{2}{*}{ Quality } \\
\hline & & & ROB & Inconsistency & Indirectness & Imprecision & $\begin{array}{l}\text { Publication } \\
\text { bias }\end{array}$ & \\
\hline $\begin{array}{l}\text { Incidence of postherpetic neuralgia at } 3 \\
\text { mo after acute herpes zoster }\end{array}$ & 26 & 3,072 & Not serious & Not serious & Serious & Not serious & Not serious & $\begin{array}{l}\oplus \oplus \oplus \bigcirc \\
\text { Moderate }\end{array}$ \\
\hline $\begin{array}{l}\text { Incidence of postherpetic neuralgia at } 1 \\
\text { mo after acute herpes zoster }\end{array}$ & 19 & 2,492 & Not serious & Not serious & Serious & Not serious & Not serious & $\begin{array}{l}\oplus \oplus \oplus \bigcirc \\
\text { Moderate }\end{array}$ \\
\hline $\begin{array}{l}\text { Incidence of postherpetic neuralgia at } 6 \\
\text { mo after acute herpes zoster }\end{array}$ & 17 & 2,502 & Not serious & Not serious & Serious & Not serious & Not serious & $\begin{array}{l}\oplus \oplus \oplus \bigcirc \\
\text { Moderate }\end{array}$ \\
\hline $\begin{array}{l}\text { Pain score measured at } 3 \text { mo after } \\
\text { acute herpes zoster }\end{array}$ & 9 & 1,225 & Not serious & Not serious & Serious & Serious & Not serious & $\begin{array}{l}\oplus \oplus \bigcirc \bigcirc \\
\text { Low }\end{array}$ \\
\hline $\begin{array}{l}\text { Pain score measured at } 1 \text { mo after } \\
\text { acute herpes zoster }\end{array}$ & 15 & 2,385 & Not serious & Not serious & Serious & Not serious & Not serious & $\begin{array}{l}\oplus \oplus \oplus \bigcirc \\
\text { Moderate }\end{array}$ \\
\hline
\end{tabular}

GRADE: Grading of Recommendations, Assessment, Development, and Evaluation, ROB: risk of bias.

+ AE/AV + AE-iLS/AV + AE-cLSC/AE-sgLS were separated, an NMA was not performed (Fig. 2F).

\section{Quality of the evidence}

Five outcomes were evaluated using the GRADE system. The evidence quality was moderate for the incidence of postherpetic neuralgia at 1,3 , and 6 months after acute $\mathrm{HZ}$ and the pain score measured at 1 month after acute HZ, whereas the evidence quality was low for pain score measured at 3 months after acute HZ (Table 3).

\section{DISCUSSION}

The present NMA demonstrated that decreasing the incidence of PHN at 3 months after acute $\mathrm{HZ}$ in the order of EPI-LSE, AV + sLS, AV + iLS, AV + AE-sgLS, and AV + AE-pV.

After the initial infection, VZV remains in a latent state inside the dorsal root ganglion. A decrease in cell-mediated immunity due to various reasons reactivates latent $\mathrm{VZV}$, thus inducing inflammatory reactions, finally leading to central and peripheral nerve damage. During these processes, unilateral and painful skin eruptions develop along the dermatome of the damaged nerve. Damaged central and peripheral nerves induce repetitive stimuli, an increase in neuronal excitability, an alteration in pain perception, and central sensitization in the nociceptive system, which subsequently leads to the development of PHN [121-123].

Our study showed that EPI-LSE was most effective in preventing PHN at 1 and 3 months after acute HZ; this finding is consistent with that of a previous systematic review, which reported that continuous epidural block is effective in preventing PHN at 3 months after acute HZ [13]. Epidural local anesthetics, steroids, and epinephrine re- duce local inflammation in the dorsal root ganglia and attenuate central sensitization, thus showing the preventive effects of EPI-LSE. Epidural analgesia also has the theoretical benefit of reducing the systemic toxicity of pharmacologic agents, because the amount of pharmacologic agent used in epidural analgesia is reduced compared with that used for systemic administration. Although many studies suggest significant benefits from epidural analgesia $[79,110]$, some studies only showed the short-term effects of this strategy in acute pain [124].

Many clinicians prescribed antiviral agents immediately after the appearance of cutaneous rash. Because viral replication induces nerve inflammation and the adjuvant tissue damage, blocking the viral replication through the administration of antiviral agents is thought to play an important role in the treatment of acute $\mathrm{HZ}$ and prevention of PHN. A previous systematic review showed that antiviral agents administered within 72 hours after the onset of skin rash may decrease the incidence or duration of PHN [12]. However, another systematic review reported that antiviral agents have no effect in reducing the incidence of PHN [125]. These differences may be because prevention of PHN can only be achieved when nerve damage and inflammatory reactions caused by viral proliferation are reduced, and central sensitization is attenuated by inhibiting the transmission of nociceptive afferent signals caused by previous damage.

In our study, the antiviral agents alone did not show a preventive effect compared with a placebo. However, antiviral agents combined with subcutaneous or intracutaneous injection of local anesthetics and steroids have shown beneficial effects. The effects of subcutaneous $[106,126]$ and intracutaneous injections $[105,107]$ have also been demonstrated in several previous studies when combined with antiviral agents. Local anesthetics injected in subcutaneous or intracutaneous lesions may block the 
C-nociceptors and the afferent transmission of painful signals. Furthermore, a steroid in the injectate decreased the neural inflammation and had a neuroprotective effect [127]. These mechanisms act synergistically to reduce pain and prevent PHN. These interventions combined with antiviral agents can be useful strategies to prevent PHN in clinical practice, because subcutaneous or intracutaneous injection is a safe, convenient, and time-efficient interventional technique.

The paravertebral block is one of the most commonly used interventions for reducing pain in patients with acute HZ. A paravertebral block is characterized by direct penetration of local anesthetics into the spinal nerve, including the dorsal ramus, rami communicants, and sympathetic chain. In our study, a paravertebral block combind with antiviral and antiepileptic agents was effective in preventing PHN at 1, 3, and 6 months; this findings is consistent with that of a previous meta-analysis, which reported the favorable impact of the paravertebral block on the prevention of PHN [13]. Although paravertebral block and epidural analgesia showed an equivalent analgesic effect for thoracotomy pain [128,129], the preventive effect of PHN in AV + AE-pV was longer (at 1, 3, and 6 months) than that in EPILSE (at 1 and 3 months). This difference may be because sympathetic blockade in the paravertebral block induces the interruption of vasospasm of endoneural arterioles and prevention of re-spasm, thereby providing a longer blocking effect and a more beneficial effect on attenuating the central sensitization $[48,102]$. However, although theoretical benefits through various mechanisms were expected, no additional benefit was shown for paravertebral blocks repeated more than twice [130]. Therefore, additional welldesigned RCTs related to the time and number of trials for effective prevention are needed. Adding antiepileptic and antiviral agents to the paravertebral block also extends the duration of preventive effects by inhibiting viral proliferation at an early stage of the neuropathic component of pain.

The present study has several limitations. First, as with all systematic reviews and meta-analyses, there was demographic, clinical, and methodological heterogeneity. However, we evaluated the transitivity assumption and performed a meta-regression analysis, which suggested the presence of heterogeneity; however, the heterogeneity had less impact on the outcomes. Second, the estimates through the NMA might be affected by inconsistencies in the NMA that compared more than two arms [131,132]. When we carefully reviewed the inconsistency in the entire network and loop to enhance the reliability of the results, overall inconsistency was less likely. The strategies determined in the current NMA were effective in a limited number of clinical trials. As our NMA was based on various single-center small-scale trials, the risk of overestimation or underestimation of the true treatment effects or lack of power to discriminate the effectiveness of strategies to prevent PHN may be present. Thus, more data from well-designed and high-quality RCTs, according to the consensus-based definition of PHN and standardized outcome assessment, are needed to verify our results.

Despite these limitations, the current NMA has several strengths compared with previous meta-analyses. First, this is the first NMA to compare and quantify the rank order of the relative effects of various strategies for preventing PHN, which may help patients, clinicians, and policy makers make evidence-based decisions when selecting strategies for preventing PHN. Second, a rigorous methodology based on a published, pre-planned protocol with a sensitive and systematic search was used. Third, inconsistencies among the enrolled studies were not significant, and the publication bias among the enrolled studies was minimal.

In summary, the continuous epidural block with local anesthetics, antiviral agents with intracutaneous or subcutaneous injection with local anesthetics and steroid, and paravertebral block combind with antiviral and antiepileptic agents are effective strategies for preventing PHN.

\section{CONFLICT OF INTEREST}

No potential conflict of interest relevant to this article was reported.

\section{FUNDING}

No funding to declare.

\section{ORCID}

Junhyeok Kim, https://orcid.org/0000-0001-6928-0723

Min Kyoung Kim, https://orcid.org/0000-0003-0477-2588

Geun Joo Choi, https://orcid.org/0000-0002-4653-4193

Hwa Yong Shin, https://orcid.org/0000-0002-8721-3070

Beom Gyu Kim, https://orcid.org/0000-0001-9166-1768

Hyun Kang, https://orcid.org/0000-0003-2844-5880

\section{SUPPLEMENTARY MATERIALS}

Supplementary materials can be found via https://doi. org/10.3344/kjp.2021.34.4.509. 


\section{REFERENCES}

1. Kawai K, Gebremeskel BG, Acosta CJ. Systematic review of incidence and complications of herpes zoster: towards a global perspective. BMJ Open 2014; 4: e004833.

2. Yawn BP, Gilden D. The global epidemiology of herpes zoster. Neurology 2013; 81: 928-30.

3. Johnson RW, Alvarez-Pasquin MJ, Bijl M, Franco E, Gaillat J, Clara JG, et al. Herpes zoster epidemiology, management, and disease and economic burden in Europe: a multidisciplinary perspective. Ther Adv Vaccines 2015; 3: 109-20.

4. Erskine N, Tran H, Levin L, Ulbricht C, Fingeroth J, Kiefe C, et al. A systematic review and meta-analysis on herpes zoster and the risk of cardiac and cerebrovascular events. PLoS One 2017; 12: e0181565.

5. Forbes HJ, Thomas SL, Smeeth L, Clayton T, Farmer R, Bhaskaran $\mathrm{K}$, et al. A systematic review and meta-analysis of risk factors for postherpetic neuralgia. Pain 2016; 157: 3054.

6. Nahm FS, Kim SH, Kim HS, Shin JW, Yoo SH, Yoon MH, et al. Survey on the treatment of postherpetic neuralgia in Korea; multicenter study of 1,414 patients. Korean J Pain 2013; 26: 21-6.

7. Sacks GM. Unmet need in the treatment of postherpetic neuralgia. Am J Manag Care 2013; 19(1 Suppl): S207-13.

8. Johnson RW, Bouhassira D, Kassianos G, Leplège A, Schmader KE, Weinke T. The impact of herpes zoster and post-herpetic neuralgia on quality-of-life. BMC Med 2010; 8: 37.

9. Schmader KE. Epidemiology and impact on quality of life of postherpetic neuralgia and painful diabetic neuropathy. Clin J Pain 2002; 18: 350-4.

10. Xing XF, Zhou ZF, Zhang FJ, Yan M. The effect of early use of supplemental therapy on preventing postherpetic neuralgia: a systematic review and meta-analysis. Pain Physician 2017; 20: 471-86

11. Han Y, Zhang J, Chen N, He L, Zhou M, Zhu C. Corticosteroids for preventing postherpetic neuralgia. Cochrane Database Syst Rev 2013; (3): CD005582.

12. Chen N, Li Q, Yang J, Zhou M, Zhou D, He L. Antiviral treatment for preventing postherpetic neuralgia. Cochrane Database Syst Rev 2014; (2): CD006866.

13. Kim HJ, Ahn HS, Lee JY, Choi SS, Cheong YS, Kwon K, et al. Effects of applying nerve blocks to prevent postherpetic neuralgia in patients with acute herpes zoster: a systematic review and meta-analysis. Korean J Pain 2017; 30: 3-17.

14. Jansen JP, Naci H. Is network meta-analysis as valid as standard pairwise meta-analysis? It all depends on the distribution of effect modifiers. BMC Med 2013; 11: 159.

15. Shamseer L, Moher D, Clarke M, Ghersi D, Liberati A, Petticrew $\mathrm{M}$, et al. Preferred reporting items for systematic review and meta-analysis protocols (PRISMA-P) 2015: elabo- ration and explanation. BMJ 2015; 350: g7647.

16. Higgins JPT, Thomas J, Chandler J, Cumpston M, Li T, Page MJ, et al. Cochrane handbook for systematic reviews of interventions. 2nd ed. Chichester, John Wiley \& Sons. 2019.

17. Cornell JE. The PRISMA extension for network metaanalysis: bringing clarity and guidance to the reporting of systematic reviews incorporating network meta-analyses. Ann Intern Med 2015; 162: 797-8.

18. Viera AJ, Garrett JM. Understanding interobserver agreement: the kappa statistic. Fam Med 2005; 37: 360-3.

19. Higgins JPT, Sterne JAC, Savović J, Page MJ, Hróbjartsson A, Boutron I, et al. A revised tool for assessing risk of bias in randomized trials. Cochrane Database Syst Rev 2016; 10 Suppl 1: 29-31.

20. Chaimani A, Higgins JP, Mavridis D, Spyridonos P, Salanti G. Graphical tools for network meta-analysis in STATA. PLoS One 2013; 8: e76654.

21. White IR, Barrett JK, Jackson D, Higgins JP. Consistency and inconsistency in network meta-analysis: model estimation using multivariate meta-regression. Res Synth Methods 2012; 3: 111-25.

22. Salanti G, Ades AE, Ioannidis JP. Graphical methods and numerical summaries for presenting results from multipletreatment meta-analysis: an overview and tutorial. J Clin Epidemiol 2011; 64: 163-71.

23. Riley RD, Higgins JP, Deeks JJ. Interpretation of random effects meta-analyses. BMJ 2011; 342: d549.

24. van Valkenhoef G, Lu G, de Brock B, Hillege H, Ades AE, Welton NJ. Automating network meta-analysis. Res Synth Methods 2012; 3: 285-99.

25. Atkins D, Best D, Briss PA, Eccles M, Falck-Ytter Y, Flottorp $\mathrm{S}$, et al. Grading quality of evidence and strength of recommendations. BMJ 2004; 328: 1490.

26. Canadian Pain Society Study Day participants. Safety and effectiveness of the herpes zoster vaccine to prevent postherpetic neuralgia: 2014 update and consensus statement from the Canadian Pain Society. Pain Res Manag 2015; 20: $46-7$.

27. Dworkin RH, Boon RJ, Griffin DR, Phung D. Postherpetic neuralgia: impact of famciclovir, age, rash severity, and acute pain in herpes zoster patients. J Infect Dis 1998; 178 Suppl 1: S76-80.

28. Dworkin RH, Schmader KE. Treatment and prevention of postherpetic neuralgia. Clin Infect Dis 2003; 36: 877-82.

29. Gawecka E, Viken O. Postherpetic neuralgia: new hopes in prevention with adult vaccination and in treatment with a concentrated capsaicin patch. Scand J Pain 2012; 3: 220-8.

30. Gilden D. Efficacy of live zoster vaccine in preventing zoster and postherpetic neuralgia. J Intern Med 2011; 269: 496-506.

31. Jackson JL, Gibbons R, Meyer G, Inouye L. The effect of treating herpes zoster with oral acyclovir in preventing postherpetic neuralgia. A meta-analysis. Arch Intern Med 
1997; 157: 909-12.

32. Wood MJ, Kay R, Dworkin RH, Soong SJ, Whitley RJ. Oral acyclovir therapy accelerates pain resolution in patients with herpes zoster: a meta-analysis of placebo-controlled trials. Clin Infect Dis 1996; 22: 341-7.

33. Wu CL, Marsh A, Dworkin RH. The role of sympathetic nerve blocks in herpes zoster and postherpetic neuralgia. Pain 2000; 87: 121-9.

34. Huff JC, Drucker JL, Clemmer A, Laskin OL, Connor JD, Bryson YJ, et al. Effect of oral acyclovir on pain resolution in herpes zoster: a reanalysis. J Med Virol 1993; Suppl 1: 93-6.

35. Surman OS, Flynn T, Schooley RT, Baer L, Parker S, Hirsch MS, et al. A double-blind, placebo-controlled study of oral acyclovir in postherpetic neuralgia. Psychosomatics 1990; 31: 287-92.

36. Whitley RJ, Weiss H, Gnann JW Jr, Tyring S, Mertz GJ, Pappas PG, et al. Acyclovir with and without prednisone for the treatment of herpes zoster. A randomized, placebo-controlled trial. The National Institute of Allergy and Infectious Diseases Collaborative Antiviral Study Group. Ann Intern Med 1996; 125: 376-83.

37. Balakrishnan S, Bhushan K, Bhargava VK, Pandhi P. A randomized parallel trial of topical aspirin-moisturizer solution vs. oral aspirin for acute herpetic neuralgia. Int J Dermatol 2001; 40: 535-8.

38. Bareggi SR, Pirola R, De Benedittis G. Skin and plasma levels of acetylsalicylic acid: a comparison between topical aspirin/diethyl ether mixture and oral aspirin in acute herpes zoster and postherpetic neuralgia. Eur J Clin Pharmacol 1998; 54: 231-5.

39. Bruni L, Tagliapietra G, Innocenti P. Herpes zoster treatments: results of a clinical trial relative to the use of rifamycin SV versus neuramide. J Int Med Res 1984; 12: 255-60.

40. Dworkin RH, Barbano RL, Tyring SK, Betts RF, McDermott MP, Pennella-Vaughan J, et al. A randomized, placebo-controlled trial of oxycodone and of gabapentin for acute pain in herpes zoster. Pain 2009; 142: 209-17.

41. Higa K, Hori K, Harasawa I, Hirata K, Dan K. High thoracic epidural block relieves acute herpetic pain involving the trigeminal and cervical regions: comparison with effects of stellate ganglion block. Reg Anesth Pain Med 1998; 23: 25-9.

42. Isbary G, Shimizu T, Zimmermann JL, Heinlin J, Al-Zaabi S, Rechfeld M, et al. Randomized placebo-controlled clinical trial showed cold atmospheric argon plasma relieved acute pain and accelerated healing in herpes zoster. Clin Plasma Med 2014; 2: 50-5.

43. Jensen-Dahm C, Rowbotham MC, Reda H, Petersen KL. Effect of a single dose of pregabalin on herpes zoster pain. Trials 2011; 12: 55.

44. Liang L, Li X, Zhang G, Sun Y, Yu H, Jiao J. Pregabalin in the treatment of herpetic neuralgia: results of a multicenter Chinese study. Pain Med 2015; 16: 160-7.
45. Lin PL, Fan SZ, Huang CH, Huang HH, Tsai MC, Lin CJ, et al. Analgesic effect of lidocaine patch $5 \%$ in the treatment of acute herpes zoster: a double-blind and vehicle-controlled study. Reg Anesth Pain Med 2008; 33: 320-5.

46. McKendrick MW, Care C, Burke C, Hickmott E, McKendrick GD. Oral acyclovir in herpes zoster. J Antimicrob Chemother 1984; 14: 661-5.

47. Mondelli M, Romano C, Passero S, Porta PD, Rossi A. Effects of acyclovir on sensory axonal neuropathy, segmental motor paresis and postherpetic neuralgia in herpes zoster patients. Eur Neurol 1996; 36: 288-92.

48. Winnie AP, Hartwell PW. Relationship between time of treatment of acute herpes zoster with sympathetic blockade and prevention of post-herpetic neuralgia: clinical support for a new theory of the mechanism by which sympathetic blockade provides therapeutic benefit. Reg Anesth 1993; 18: 277-82.

49. Hui F, Boyle E, Vayda E, Glazier RH. A randomized controlled trial of a multifaceted integrated complementaryalternative therapy for chronic herpes zoster-related pain. Altern Med Rev 2012; 17: 57-68.

50. Juel-Jensen BE, Khan JA, Pasvol G. High-dose intravenous acyclovir in the treatment of zoster: a double-blind, placebo-controlled trial. J Infect 1983; 6(1 Suppl): 31-6.

51. Kikuchi A, Kotani N, Sato T, Takamura K, Sakai I, Matsuki A. Comparative therapeutic evaluation of intrathecal versus epidural methylprednisolone for long-term analgesia in patients with intractable postherpetic neuralgia. Reg Anesth Pain Med 1999; 24: 287-93.

52. van den Broek PJ, van der Meer JW, Mulder JD, Versteeg J, Mattie H. Limited value of acyclovir in the treatment of uncomplicated herpes zoster: a placebo-controlled study. Infection 1984; 12: 338-41.

53. Beutner KR, Friedman DJ, Forszpaniak C, Andersen PL, Wood MJ. Valaciclovir compared with acyclovir for improved therapy for herpes zoster in immunocompetent adults. Antimicrob Agents Chemother 1995; 39: 1546-53.

54. Yanagida H, Suwa K, Corssen G. No prophylactic effect of early sympathetic blockade on postherpetic neuralgia. Anesthesiology 1987; 66: 73-6.

55. Sederholm B, Peterson D. Zoster vaccine to prevent postherpetic neuralgia. J Pain Palliat Care Pharmacother 2006; 20: 41-2.

56. Dai J, Yin AH, Zhou Y, Yin LJ. [Herpes zoster treated with meridian-collateral electric information therapy combined with pricking and cupping]. Zhongguo Zhen Jiu 2011; 31: 416-9. Chinese.

57. Fujii H, Fukushima T, Ishii M, Nagano Y, Kawanishi S, Watanabe $Y$, et al. [The efficacy of intravenous lidocaine for acute herpetic pain--placebo controlled trial]. Masui 2009; 58: 1413-7. Japanese.

58. Galbraith AW. Prevention of post-herpetic neuralgia by 
amantadine hydrochloride (Symmetrel). Br J Clin Pract 1983; 37: 304-6.

59. Morton P, Thomson AN. Oral acyclovir in the treatment of herpes zoster in general practice. N Z Med J 1989; 102: 93-5.

60. Peng Z, Wang S, Huang X, Xiao P. Effect of hyperbaric oxygen therapy on patients with herpes zoster. Undersea Hyperb Med 2012; 39: 1083-7.

61. Varotti C, Rafanelli A. Evaluation of efficacy and tolerance of neuramide in the treatment of herpes zoster and postherpetic neuritis. Drugs Exp Clin Res 2001; 27: 199-208.

62. Wood MJ, Ogan PH, McKendrick MW, Care CD, McGill JI, Webb EM. Efficacy of oral acyclovir treatment of acute herpes zoster. Am J Med 1988; 85(2A): 79-83.

63. Harding SP, Lipton JR, Wells JC. Natural history of herpes zoster ophthalmicus: predictors of postherpetic neuralgia and ocular involvement. Br J Ophthalmol 1987; 71: 353-8.

64. Barbarisi M, Pace MC, Passavanti MB, Maisto M, Mazzariello L, Pota V, et al. Pregabalin and transcutaneous electrical nerve stimulation for postherpetic neuralgia treatment. Clin J Pain 2010; 26: 567-72.

65. Guo W, Xiao Z, Yang Y. Effectiveness of transdermal fentanyl combined with clodine for pain control of acute herpes zoster. J Dalian Med Univ 2007; 29: 255-6.

66. Tenicela R, Lovasik D, Eaglstein W. Treatment of herpes zoster with sympathetic blocks. Clin J Pain 1985; 1: 63-8.

67. Wang JS. The comparison of therapeutic effect of three methods for the treatment of acute herpes zoster. Curr Opin Clin Exp Res 2002; 4: 12-5.

68. Huff JC, Bean B, Balfour HH Jr, Laskin OL, Connor JD, Corey $\mathrm{L}$, et al. Therapy of herpes zoster with oral acyclovir. Am J Med 1988; 85(2A): 84-9.

69. McKendrick MW, McGill JI, White JE, Wood MJ. Oral acyclovir in acute herpes zoster. Br Med J (Clin Res Ed) 1986; 293: 1529-32.

70. Opstelten W, van Wijck AJ, Moons KG, van Essen GA, Stolker RJ, Kalkman CJ, et al. [Treatment of patients with herpes zoster by epidural injection of steroids and local anaesthetics: less pain after 1 month, but no effect on long-term postherpetic neuralgia--a randomised trial]. Ned Tijdschr Geneeskd 2006; 150: 2649-55. Dutch.

71. Peterslund NA, Seyer-Hansen K, Ipsen J, Esmann V, Schonheyder H, Juhl H. Acyclovir in herpes zoster. Lancet 1981; 2: 827-30.

72. Colin J, Prisant O, Cochener B, Lescale O, Rolland B, HoangXuan T. Comparison of the efficacy and safety of valaciclovir and acyclovir for the treatment of herpes zoster ophthalmicus. Ophthalmology 2000; 107: 1507-11.

73. Fujiwara A, Watanabe K, Hashizume K, Shinohara K, Kawaguchi M. Transforaminal vs interlaminar epidural steroid injection for acute-phase shingles: a randomized, prospective trial. Pain Physician 2018; 21: 373-82.

74. Hoang-Xuan T, Büchi ER, Herbort CP, Denis J, Frot P, Thé- nault S, et al. Oral acyclovir for herpes zoster ophthalmicus. Ophthalmology 1992; 99: 1062-70.

75. Tyring SK, Beutner KR, Tucker BA, Anderson WC, Crooks RJ. Antiviral therapy for herpes zoster: randomized, controlled clinical trial of valacyclovir and famciclovir therapy in immunocompetent patients 50 years and older. Arch Fam Med 2000; 9: 863-9.

76. Sharif S, Aslam F, Faisal LR, Saleem MA. Comparison of efficacy of two different doses of famciclovir in the prevention and treatment of postherpetic neuralgia. J Ayub Med Coll Abbottabad 2019; 31(4 Suppl 1): S668-71.

77. Tyring SK, Lee P, Hill GT Jr, Silverfield JC, Moore AY, Matkovits $\mathrm{T}$, et al. FV-100 versus valacyclovir for the prevention of post-herpetic neuralgia and the treatment of acute herpes zoster-associated pain: a randomized-controlled trial. J Med Virol 2017; 89: 1255-64.

78. Finn R, Smith MA. Oral acyclovir for herpes zoster. Lancet 1984; 2: 575.

79. Hwang SM, Kang YC, Lee YB, Yoon KB, Ahn SK, Choi EH. The effects of epidural blockade on the acute pain in herpes zoster. Arch Dermatol 1999; 135: 1359-64.

80. Malin JP. A retrospective and an observational study with acyclovir. J Med Virol 1993; Suppl 1: 102-5.

81. Colding A. Treatment of pain: organization of a pain clinic: treatment of acute herpes zoster. Proc R Soc Med 1973; 66: 541-3.

82. Colding A. The effect of regional sympathetic blocks in the treatment of herpes zoster: a survey of 300 cases. Acta Anaesthesiol Scand 1969; 13: 133-41.

83. Bean B, Braun C, Balfour HH Jr. Acyclovir therapy for acute herpes zoster. Lancet 1982; 2: 118-21.

84. Esmann V, Ipsen J, Peterslund NA, Seyer-Hansen K, Schønheyder H, Juhl H. Therapy of acute herpes zoster with acyclovir in the nonimmunocompromised host. Am J Med 1982; 73(1A): 320-5.

85. Balfour HH Jr, Bean B, Laskin OL, Ambinder RF, Meyers JD, Wade JC, et al. Acyclovir halts progression of herpes zoster in immunocompromised patients. N Engl J Med 1983; 308: 1448-53.

86. McGill J, MacDonald DR, Fall C, McKendrick GD, Copplestone A. Intravenous acyclovir in acute herpes zoster infection. J Infect 1983; 6: 157-61.

87. Cobo LM, Foulks GN, Liesegang T, Lass J, Sutphin JE, Wilhelmus K, et al. Oral acyclovir in the treatment of acute herpes zoster ophthalmicus. Ophthalmology 1986; 93: 763-70.

88. Esmann V, Geil JP, Kroon S, Fogh H, Peterslund NA, Petersen CS, et al. Prednisolone does not prevent post-herpetic neuralgia. Lancet 1987; 2: 126-9.

89. Wassilew SW, Reimlinger S, Nasemann T, Jones D. Oral acyclovir for herpes zoster: a double-blind controlled trial in normal subjects. Br J Dermatol 1987; 117: 495-501.

90. Mandal BK, Dunbar EM, Ellis ME, Ellis J, Dowd P. A double- 
masked, placebo-controlled trial of acyclovir cream in immunocompetent patients with herpes zoster. J Infect 1988; 17: 57-63.

91. Payne CM, Menday AP, Rogers T, Staughton RC. Isoprinosine does not influence the natural history of herpes zoster or postherpetic neuralgia. Scand J Infect Dis 1989; 21: 15-8.

92. Benoldi D, Mirizzi S, Zucchi A, Allegra F. Prevention of post-herpetic neuralgia. Evaluation of treatment with oral prednisone, oral acyclovir, and radiotherapy. Int J Dermatol 1991; 30: 288-90.

93. Bowsher D. The effects of pre-emptive treatment of postherpetic neuralgia with amitriptyline: a randomized, doubleblind, placebo-controlled trial. J Pain Symptom Manage 1997; 13: 327-31.

94. Ahmed HE, Craig WF, White PF, Ghoname ES, Hamza MA, Gajraj NM, et al. Percutaneous electrical nerve stimulation: an alternative to antiviral drugs for acute herpes zoster. Anesth Analg 1998; 87: 911-4.

95. Lee IH, Kim BS, Lee SC, Jo DH. The effect of stellate ganglion block on the pain of acute stage and the prevention of postherpetic neuralgin in the treatment of senile herpes zoster patients. Korean J Dermatol 1999; 37: 571-9.

96. van Wijck AJ, Opstelten W, Moons KG, van Essen GA, Stolker RJ, Kalkman CJ, et al. The PINE study of epidural steroids and local anaesthetics to prevent postherpetic neuralgia: a randomised controlled trial. Lancet 2006; 367: 219-24.

97. Ji G, Niu J, Shi Y, Hou L, Lu Y, Xiong L. The effectiveness of repetitive paravertebral injections with local anesthetics and steroids for the prevention of postherpetic neuralgia in patients with acute herpes zoster. Anesth Analg 2009; 109: 1651-5.

98. Krcevski Skvarc N, Kamenik M. Effects of pregabalin on acute herpetic pain and postherpetic neuralgia incidence. Wien Klin Wochenschr 2010; 122 Suppl 2: 49-53.

99. Kanodia SK, Singhal KC. A study on efficacy of Pregabalin in acute Herpetic Neuralgia. Ann Neurosci 2011; 18: 148-50.

100. Kanodia SK, Seth AK, Dixit AM. Dose related efficacy of gabapentin in acute herpetic neuralgia among geriatric patients. Indian J Dermatol 2012; 57: 362-5.

101. Makharita MY, Amr YM, El-Bayoumy Y. Effect of early stellate ganglion blockade for facial pain from acute herpes zoster and incidence of postherpetic neuralgia. Pain Physician 2012; 15: 467-74.

102. Makharita MY, Amr YM, El-Bayoumy Y. Single paravertebral injection for acute thoracic herpes zoster: a randomized controlled trial. Pain Pract 2015; 15: 229-35.

103. Stepanović A, Kolšek M, Kersnik J, Erčulj V. Prevention of post-herpetic neuralgia using transcutaneous electrical nerve stimulation. Wien Klin Wochenschr 2015; 127: 369-74.

104. Lee EG, Lee HJ, Hyun DJ, Min K, Kim DH, Yoon MS. Efficacy of low dose gabapentin in acute herpes zoster for preventing postherpetic neuralgia: a prospective controlled study. Der- matol Ther 2016; 29: 184-90.

105. Cui JZ, Zhang XB, Zhu P, Zhao ZB, Geng ZS, Zhang YH, et al. Effect of repetitive intracutaneous injections with local anesthetics and steroids for acute thoracic herpes zoster and incidence of postherpetic neuralgia. Pain Med 2017; 18: 1566-72.

106. Ni J, Wang X, Tang Y, Yang L, Zeng Y, Guo Y. Subcutaneous injection of triamcinolone and lidocaine to prevent postherpetic neuralgia. Pain Physician 2017; 20: 397-403.

107. Cui JZ, Zhang JW, Yan F, Yang XN, Wang XL, Zhao ZB, et al. Effect of single intra-cutaneous injection for acute thoracic herpes zoster and incidence of postherpetic neuralgia. Pain Manag Nurs 2018; 19: 186-94.

108. Bulilete O, Leiva A, Rullán M, Roca A, Llobera J; PHN Group. Efficacy of gabapentin for the prevention of postherpetic neuralgia in patients with acute herpes zoster: a double blind, randomized controlled trial. PLoS One 2019; 14: e0217335.

109. Zheng S, Li X, Yang X, He L, Xue Y, Yang Z. Ultrasoundguided cervical nerve root block for the treatment of acute cervical herpes zoster: a randomized controlled clinical study. Pain Pract 2019; 19: 500-9.

110. Pasqualucci A, Pasqualucci V, Galla F, De Angelis V, Marzocchi V, Colussi R, et al. Prevention of post-herpetic neuralgia: acyclovir and prednisolone versus epidural local anesthetic and methylprednisolone. Acta Anaesthesiol Scand 2000; 44: 910-8.

111. Wood MJ, McKendrick MW, Freris MW, Jeal SC, Jones DA, Gilbert AM. Trough plasma acyclovir concentrations and safety of oral acyclovir, $800 \mathrm{mg}$ five times daily for 7 days in elderly patients with herpes zoster. J Antimicrob Chemother 1994; 33: 1245-9.

112. Clemmensen OJ, Andersen KE. ACTH versus prednisone and placebo in herpes zoster treatment. Clin Exp Dermatol 1984; 9: 557-63.

113. Eaglstein WH, Katz R, Brown JA. The effects of early corticosteroid therapy on the skin eruption and pain of herpes zoster. JAMA 1970; 211: 1681-3.

114. Harding SP, Porter SM. Oral acyclovir in herpes zoster ophthalmicus. Curr Eye Res 1991; 10 Suppl: 177-82.

115. Keczkes K, Basheer AM. Do corticosteroids prevent postherpetic neuralgia? Br J Dermatol 1980; 102: 551-5.

116. Hügler P, Siebrecht P, Hoffmann K, Stücker M, Windeler J, Altmeyer $\mathrm{P}$, et al. Prevention of postherpetic neuralgia with varicella-zoster hyperimmune globulin. Eur J Pain 2002; 6: 435-45.

117. Cui JZ, Zhang JW, Zhang Y, Ma ZL. [Efficacy of intracutaneous methylene blue injection for moderate to severe acute thoracic herpes zoster pain and prevention of postherpetic neuralgia in elderly patients]. Nan Fang Yi Ke Da Xue Xue Bao 2016; 36: 1377-81. Chinese.

118. Manabe H, Dan K, Hirata K, Hori K, Shono S, Tateshi S, et al. 
Optimum pain relief with continuous epidural infusion of local anesthetics shortens the duration of zoster-associated pain. Clin J Pain 2004; 20: 302-8.

119. Harding SP, Lipton JR, Wells JC, Campbell JA. Relief of acute pain in herpes zoster ophthalmicus by stellate ganglion block. Br Med J (Clin Res Ed) 1986; 292: 1428.

120. Wan C, Dong DS, Song T. High-voltage, long-duration pulsed radiofrequency on Gasserian ganglion improves acute/subacute zoster-related trigeminal neuralgia: a randomized, double-blinded, controlled trial. Pain Physician 2019; 22: 361-8.

121. Arvin AM. Varicella-zoster virus. Clin Microbiol Rev 1996; 9: 361-81.

122. Harpaz R, Nagel MA, Schmader K, Tyring SK, Yawn BP. Roundtable on postherpetic neuralgia--what, why, how long, and what's next? Popul Health Manag 2012; 15: 385-90.

123. Woolf CJ. A new strategy for the treatment of inflammatory pain. Prevention or elimination of central sensitization. Drugs 1994; 47 Suppl 5: 1-9.

124. Riopelle JM, Naraghi M, Grush KP. Chronic neuralgia incidence following local anesthetic therapy for herpes zoster. Arch Dermatol 1984; 120: 747-50.

125. Li Q, Chen N, Yang J, Zhou M, Zhou D, Zhang Q, et al. Antiviral treatment for preventing postherpetic neuralgia. Cochrane Database Syst Rev 2009; (2): CD006866.
126. Epstein E. Treatment of herpes zoster and postzoster neuralgia by subcutaneous injection of triamcinolone. Int J Dermatol 1981; 20: 65-8.

127. Johansson A, Bennett GJ. Effect of local methylprednisolone on pain in a nerve injury model. A pilot study. Reg Anesth 1997; 22: 59-65.

128. Davies RG, Myles PS, Graham JM. A comparison of the analgesic efficacy and side-effects of paravertebral vs epidural blockade for thoracotomy--a systematic review and metaanalysis of randomized trials. Br J Anaesth 2006; 96: 418-26.

129. Joshi GP, Bonnet F, Shah R, Wilkinson RC, Camu F, Fischer $\mathrm{B}$, et al. A systematic review of randomized trials evaluating regional techniques for postthoracotomy analgesia. Anesth Analg 2008; 107: 1026-40.

130. Makharita MY, Amr YM. Effect of repeated paravertebral injections with local anesthetics and steroids on prevention of post-herpetic neuralgia. Pain Physician 2020; 23: 565-72.

131. Stettler C, Wandel S, Allemann S, Kastrati A, Morice MC, Schömig A, et al. Outcomes associated with drug-eluting and bare-metal stents: a collaborative network meta-analysis. Lancet 2007; 370: 937-48.

132. Li T, Puhan MA, Vedula SS, Singh S, Dickersin K. Network meta-analysis-highly attractive but more methodological research is needed. BMC Med 2011; 9: 79. 


\section{Appendix. Search term}

\section{MEDLINE}

1. randomized controlled trial.pt

2. randomized controlled trial\$.mp

3. controlled clinical trial.pt

4. controlled clinical trial\$.mp

5. random allocation.mp

6. exp double-blind method/

7. double-blind.mp

8. exp single-blind method/

9. $\quad$ single-blind.mp

10. or/1-9

11. clinical trial.pt

12. clinical trial\$.mp

13. exp clinical trial/

14. (clin\$ adj25 trial\$).mp

15. ((singl\$ or doubl\$ or tripl\$ or trebl\$) adj25 (blind $\$$ or mask $\$)$ ).mp

16. random $\$ . m p$

17. exp research design/

18. research design.mp

19. or/11-18

20. 10 or 19

21. Case report.tw.

22. Letter.pt.

23. Historical article.pt.

24. Review.pt.

25. or/21-24

26. 20 not 25

27. Exp Neuralgia, Postherpetic/

28. Zoster-associated pain.mp

29. Herpetic pain.mp

30. Herpetic Neuralgia.mp

31. Postherpetic neuralgia.mp

32. PHN.mp

33. or/27-32

34. Herpes zoster.mp

35. Herpes \$.mp

36. herpetic $\$ . m p$

37. HHV.mp

38. Zona.mp

39. Zoster.mp

40. Shingles.mp

41. Varicella.mp

42. Chickenpox.mp

43. post-herpetic.mp

44. VZV.mp

45. Or 34-44/

46. neuralgi\$.mp

47. pain.mp

48. or $46-47 /$

49. $\quad 45$ and 48 
50. 33 or 49

51. 26 and 50

52. Exp Antidepressive agents/

53. Amitriptyline.mp

54. (Damilen or Domical or Tryptine or Tryptizol or Tryptanol or Elavil or Amineurin or Amitrip or Laroxyl or Endep or Lentizol or Novoprotect or Saroten or Syneudon or Triptafen or Amitrol or Anapsique or Amitriptylin\$).ti,ab,hw.

55. Norttriptyline.mp

56. (Allegron or Aventyl or Noritren or Norpress or Nortrilen or Norventyl or Norzepine or Pamelor or Sensoval) .ti,ab,hw.

57. Imipramine.mp

58. (Tofranil orTofranil-PM or Imiprami\$ or imizine).ti,ab,hw.

59. Milnacipran.mp

60. (Ixel or Toledomin or Dalcipran or savella or impulsor).ti,ab,hw.

61. Or $52-60 /$

62. Exp anticonvulsants/

63. Gabapentin.mp

64. (Gabapentin or Neurontin).ti,ab,hw.

65. Pregabalin.mp

66. (Pregabalin or Lyrica).ti,ab,hw.

67. Carbamazepine.mp

68. (Carbamazepin $\$$ or Neurotol or Tegretol or Amizepine or Epitol or Carbazepin or Finlepsin).ti,ab,hw.

69. Oxycarbamazepine.mp

70. (oxycarbazep\$ or OCBZ or Oxtellar or Trileptal).ti,ab,hw

71. OR 62-70/

72. Exp Capsaicin/

73. (Capsaicin\$ or Nonenamide or Axsain or Zacin or Capsicum or Capsidol or Zostrix or Capzasin or Gelcen or Katrum or Capsin).ti,ab,hw.

74. Exp Botulinum Toxins/

75. (Jeuveau or Botox or Xeomin or Myobloc or Neuronox or BTX or Neurobloc).ti,ab,hw.

76. Exp analgesics/

77. Exp analgesics, opioid/

78. (Morphine or Fentanyl or Alfentanil or Sufentanil or Remifentanil or Buprenorphine or Meperidine or Pethidine or Nalbuphine or pentazocin or butophanol or nalorphine or oxycodone or hydromorphone or oxynorm or methadone).ti,ab,hw.

79. Exp Anti-Inflammatory Agents, Non-Steroidal/

80. (aspirin or diclofenac or Cambia or Cataflam or Voltaren-XR or Zipsor or Zorvolex or diflunisal or Dolobid or etodolac or ibuprofen or Motrin or Advil or indomethacin or Indocin or ketoprofen or Active-Ketoprofen or Orudis or ketorolac or Toradol or nabumetone or Relafen or naproxen or Aleve or Anaprox or Naprelan or Naprosyn or oxaprozin or Daypro or piroxicam or Feldene or salsalate or Disalsate or Amigesic or sulindac or Clinoril or tolmetin or Tolectin).ti,ab,hw.

81. Exp Cyclooxygenase 2 Inhibitors/

82. (cox 2 or Celecoxib or Consensi or valdecoxib or Bextra or Rofecoxib or Vioxx or Celebrex or Etoricoxib).ti,ab,hw.

83. Exp acetaminophen/

84. (Paracetamol or APAP or acetyl-para-aminophenol or Actamin or Anacin AF or Apra or Bromo Seltzer or Children's Tylenol or Elixsure Fever/Pain or Mapap or Medi-Tabs or Q-Pap or Silapap Childrens or Tactinal or Tempra Quicklets or Tycolene or Tylenol or Vitapap).ti,ab,hw.

85. Exp Lidocaine/

86. (Lidocaine or Lignocaine or Xyloneural or Octocaine or Xylesthesin or Xylocaine or Xylocitin or Dalcaine or Versatis).ti,ab,hw.

87. Exp Ketamine/

88. (Ketamin or Ketamina or Ketamine or Ketaminol or Ketanest or Ketaset or Tekam or Vetalar or Ketalar or Calipsol or Kalipsol or Calypsol).ti,ab,hw. 
89. Exp clonidine/

90. (Klofenil or Clofenil or Chlophazolin or ST-155 or ST 155 or ST155 or Gemiton or Hemiton or Isoglaucon or Klofelin or Clofelin or Clopheline or M-5041T or M 5041T or M5041T or Catapres or Catapresan or Catapressan or Dixarit). ti,ab,hw.

91. Exp Dexmedetomidine/

92. (Dexmedetomidin\$ or MPV-1440 or MPV 1440 or MPV1440 or Precedex or MPV 785).ti,ab,hw.

93. Exp Vitamin B 12/

94. (Vitamin B12 or Cyanocobalamin or Cobalamins or Cobalamin or Eritron).ti,ab,hw.

95. Or 52-94/

96. Exp Nerve Block/

97. Nerve.mp

98. Block\$.mp

99. 97 and 98

100. Chemical.mp

101. Neurolys $\$ . m p$

102. 100 and 101

103. Chemodenervation\$.mp

104. 96 or 99 or 102 or 103

105. Neuraxial block.mp

106. Exp Anesthesia, Epidural/

107. Epidural block.mp

108. Paravertebral block.mp

109. stellate ganglion block.mp

110. local injection.mp

111. Exp Transcutaneous Electric Nerve Stimulation/

112. Nerve stimulation.mp

113. Exp Pulsed Radiofrequency Treatment/

114. Heat RF.mp

115. Exp spinal cord stimulation/

116. Exp Electroacupuncture/

117. Destruction and (dorsal root ganglion or drg).mp

118. Nerve destruction.mp

119. Exp Acupuncture/

120. (acupuncture or needle or needling or electro-acupuncture or cupping or moxibustion or pricking or pyonex or bloodletting).mp

121. Exp Anesthesia, Local/

122. OR $96-121 /$

123. 95 OR 122

124. 51 AND 123 